\title{
Reflection factorizations of Singer cycles
}

\author{
J. B. Lewis - V. Reiner - D. Stanton
}

Received: 03 September 2013 / Accepted: 15 January 2014 / Published online: 25 March 2014

(C) Springer Science+Business Media New York 2014

\begin{abstract}
The number of shortest factorizations into reflections for a Singer cycle in $\mathrm{GL}_{n}\left(\mathbb{F}_{q}\right)$ is shown to be $\left(q^{n}-1\right)^{n-1}$. Formulas counting factorizations of any length, and counting those with reflections of fixed conjugacy classes are also given. The method is a standard character-theory technique, requiring the compilation of irreducible character values for Singer cycles, semisimple reflections, and transvections. The results suggest several open problems and questions, which are discussed at the end.
\end{abstract}

Keywords Singer cycle $\cdot$ Reflection $\cdot$ Transvection $\cdot$ Factorization

\section{Introduction and main result}

This paper was motivated by two classic results on the number $t(n, \ell)$ of ordered factorizations $\left(t_{1}, \ldots, t_{\ell}\right)$ of an $n$-cycle $c=t_{1} t_{2} \cdots t_{\ell}$ in the symmetric group $\mathfrak{S}_{n}$, where each $t_{i}$ is a transposition.

Theorem (Hurwitz [14], Dénes [6]) For $n \geq 1$, one has $t(n, n-1)=n^{n-2}$.

\footnotetext{
J. B. Lewis $(\varangle) \cdot$ V. Reiner $\cdot$ D. Stanton

School of Mathematics, University of Minnesota, Minneapolis, MN 55455, USA

e-mail: jblewis@math.umn.edu

V. Reiner

e-mail: reiner@math.umn.edu

D. Stanton

e-mail: stanton@math.umn.edu
} 
Theorem (Jackson [16, p. 368]) For $n \geq 1$, more generally $t(n, \ell)$ has ordinary generating function

$$
\sum_{\ell \geq 0} t(n, \ell) x^{\ell}=n^{n-2} x^{n-1} \prod_{k=0}^{n-1}\left(1-x n\left(\frac{n-1}{2}-k\right)\right)^{-1}
$$

and explicit formulas

$$
\begin{aligned}
t(n, \ell) & =\frac{n^{\ell}}{n !} \sum_{k=0}^{n-1}(-1)^{k}\left(\begin{array}{c}
n-1 \\
k
\end{array}\right)\left(\frac{n-1}{2}-k\right)^{\ell} \\
& =\frac{(-n)^{\ell}}{n !}(-1)^{n-1}\left[\Delta^{n-1}\left(x^{\ell}\right)\right]_{x=\frac{1-n}{2}}
\end{aligned}
$$

Here, the difference operator $\Delta(f)(x):=f(x+1)-f(x)$ satisfies $\Delta^{n}(f)(x):=$ $\sum_{k=0}^{n}(-1)^{n-k}\left(\begin{array}{l}n \\ k\end{array}\right) f(x+k)$.

Our goals are $q$-analogs, replacing the symmetric group $\mathfrak{S}_{n}$ with the general linear group $\mathrm{GL}_{n}\left(\mathbb{F}_{q}\right)$, replacing transpositions with reflections, and replacing an $n$-cycle with a Singer cycle $c$ : the image of a generator for the cyclic group $\mathbb{F}_{q^{n}}^{\times} \cong \mathbb{Z} /\left(q^{n}-1\right) \mathbb{Z}$ under any embedding $\mathbb{F}_{q^{n}}^{\times} \hookrightarrow \mathrm{GL}_{\mathbb{F}_{q}}\left(\mathbb{F}_{q^{n}}\right) \cong \mathrm{GL}_{n}\left(\mathbb{F}_{q}\right)$ that comes from a choice of $\mathbb{F}_{q^{-}}$ vector space isomorphism $\mathbb{F}_{q^{n}} \cong \mathbb{F}_{q}^{n}$. The analogy between Singer cycles in $\operatorname{GL}_{n}\left(\mathbb{F}_{q}\right)$ and $n$-cycles in $\mathfrak{S}_{n}$ is reasonably well established [25, §7], [26, §§8-9]. Fixing such a Singer cycle $c$, denote by $t_{q}(n, \ell)$ the number of ordered factorizations $\left(t_{1}, \ldots, t_{\ell}\right)$ of $c=t_{1} t_{2} \cdots t_{\ell}$ in which each $t_{i}$ is a reflection in $\mathrm{GL}_{n}\left(\mathbb{F}_{q}\right)$, that is, the fixed space $\left(\mathbb{F}_{q}^{n}\right)^{t_{i}}$ is a hyperplane in $\mathbb{F}_{q}^{n}$.

Theorem 1.1 For $n \geq 2$, one has $t_{q}(n, n)=\left(q^{n}-1\right)^{n-1}$.

Theorem 1.2 For $n \geq 2$, more generally, $t_{q}(n, \ell)$ has ordinary generating function:

$$
\sum_{\ell \geq 0} t_{q}(n, \ell) x^{\ell}=\left(q^{n}-1\right)^{n-1} x^{n} \cdot\left(1+x[n]_{q}\right)^{-1} \prod_{k=0}^{n-1}\left(1+x[n]_{q}\left(1+q^{k}-q^{k+1}\right)\right)^{-1}
$$

and explicit formulas:

$$
\begin{aligned}
t_{q}(n, \ell)= & \frac{\left(-[n]_{q}\right)^{\ell}}{q^{\left(\begin{array}{c}
n \\
2
\end{array}\right)}(q ; q)_{n}}\left((-1)^{n-1}(q ; q)_{n-1}+\sum_{k=0}^{n-1}(-1)^{k+n}\right. \\
& \left.q^{\left(\begin{array}{c}
k+1 \\
2
\end{array}\right)}\left[\begin{array}{c}
n-1 \\
k
\end{array}\right]_{q}\left(1+q^{n-k-1}-q^{n-k}\right)^{\ell}\right) \\
= & (1-q)^{-1} \frac{\left(-[n]_{q}\right)^{\ell}}{[n] !_{q}}\left[\Delta_{q}^{n-1}\left(\frac{1}{x}-\frac{(1+x(1-q))^{\ell}}{x}\right)\right]_{x=1} \\
= & {[n]_{q}^{\ell-1} \sum_{i=0}^{\ell-n}(-1)^{i}(q-1)^{\ell-i-1}\left(\begin{array}{c}
\ell \\
i
\end{array}\right)\left[\begin{array}{c}
\ell-i-1 \\
n-1
\end{array}\right]_{q} . }
\end{aligned}
$$


The $q$-analogs used above and elsewhere in the paper are defined as follows:

$$
\begin{aligned}
& {\left[\begin{array}{l}
n \\
k
\end{array}\right]_{q}:=\frac{[n] !_{q}}{[k] !_{q}[n-k] !_{q}} \text {, where }[n] !_{q}:=[1]_{q}[2]_{q} \cdots[n]_{q} \text { and }} \\
& {[n]_{q}:=1+q+q^{2}+\cdots+q^{n-1} \text {, }} \\
& (x ; q)_{n}:=(1-x)(1-x q)\left(1-x q^{2}\right) \cdots\left(1-x q^{n-1}\right) \text {, and } \\
& \Delta_{q}(f)(x):=\frac{f(x)-f(q x)}{x-q x} \text {, so that } \\
& \Delta_{q}^{n}(f)(x)=\frac{1}{q^{\left(\begin{array}{c}
n \\
2
\end{array}\right)} x^{n}(1-q)^{n}} \sum_{k=0}^{n}(-1)^{n-k} q^{\left(\begin{array}{c}
k \\
2
\end{array}\right)}\left[\begin{array}{l}
n \\
k
\end{array}\right]_{q} f\left(q^{n-k} x\right) .
\end{aligned}
$$

The equivalence of the three formulas (1.4), (1.5), (1.6) for $t_{q}(n, \ell)$ is explained in Proposition 5.1.

In fact, we will prove the following refinement of Theorem 1.2 for $q>2$, having no counterpart for $\mathfrak{S}_{n}$. Transpositions are all conjugate within $\mathfrak{S}_{n}$, but the conjugacy class of a reflection $t$ in $\mathrm{GL}_{n}\left(\mathbb{F}_{q}\right)$ for $q>2$ varies with its determinant $\operatorname{det}(t)$ in $\mathbb{F}_{q}^{\times}$. When $\operatorname{det}(t)=1$, the reflection $t$ is called a transvection [20, XIII §9], while $\operatorname{det}(t) \neq 1$ means that $t$ is a semisimple reflection. One can associate to an ordered factorization $\left(t_{1}, \ldots, t_{\ell}\right)$ of $c=t_{1} t_{2} \cdots t_{\ell}$ the sequence of determinants $\left(\operatorname{det}\left(t_{1}\right), \ldots, \operatorname{det}\left(t_{\ell}\right)\right)$ in $\mathbb{F}_{q}^{\ell}$, having product $\operatorname{det}(c)$.

Theorem 1.3 Let $q>2$. Fix a Singer cycle c in $\mathrm{GL}_{n}\left(\mathbb{F}_{q}\right)$ and a sequence $\alpha=\left(\alpha_{i}\right)_{i=1}^{\ell}$ in $\left(\mathbb{F}_{q}^{\times}\right)^{\ell}$ with $\prod_{i=1}^{\ell} \alpha_{i}=\operatorname{det}(c)$. Let $m$ be the number of values $i$ such that $\alpha_{i}=1$. Then, one has $m \leq \ell-1$, and the number of ordered reflection factorizations $c=$ $t_{1} \cdots t_{\ell}$ with $\operatorname{det}\left(t_{i}\right)=\alpha_{i}$ depends only upon $\ell$ and $m$. This quantity $t_{q}(n, \ell, m)$ is given by these formulas:

$$
\begin{aligned}
t_{q}(n, \ell, m) & =[n]_{q}^{\ell-1} \sum_{i=0}^{\min (m, \ell-n)}(-1)^{i}\left(\begin{array}{c}
m \\
i
\end{array}\right)\left[\begin{array}{c}
\ell-i-1 \\
n-1
\end{array}\right]_{q} \\
& =\frac{[n]_{q}^{\ell}}{[n] !_{q}}\left[\Delta_{q}^{n-1}\left((x-1)^{m} x^{\ell-m-1}\right)\right]_{x=1} .
\end{aligned}
$$

In particular, setting $\ell=n$ in (1.8), the number of shortest such factorizations is

$$
t_{q}(n, n, m)=[n]_{q}^{n-1}
$$

which depends neither on the sequence $\alpha=\left(\operatorname{det}\left(t_{i}\right)\right)_{i=1}^{\ell}$ nor on the number of transvections $m$.

The equivalence of the formulas (1.8) and (1.9) for $t_{q}(n, \ell, m)$ is also explained in Proposition 5.1 below.

Theorems 1.2 and 1.3 are proven via a standard character-theoretic approach. This approach is reviewed quickly in Sect. 2, followed by an outline of ordinary character 
theory for $\mathrm{GL}_{n}\left(\mathbb{F}_{q}\right)$ in Sect. 3. Section 4 either reviews or derives the needed explicit character values for four kinds of conjugacy classes: the identity element, Singer cycles, semisimple reflections, and transvections. Then, Sect. 5 assembles these calculations into the proofs of Theorems 1.2 and 1.3. Section 6 closes with some further remarks and questions.

Although Theorem 1.3 is stated for $q>2$, something interesting also occurs for $q=2$. All reflections in $\mathrm{GL}_{n}\left(\mathbb{F}_{2}\right)$ are transvections; thus, one always has $m=\ell$ for $q=2$. Furthermore, one can see that (1.6), (1.8) give the same answer when both $q=2$ and $m=\ell$. This reflects a striking dichotomy in our proofs: for $q>2$ the only contributions to the computation come from irreducible characters of $\mathrm{GL}_{n}\left(\mathbb{F}_{q}\right)$ arising as constituents of parabolic inductions of characters of $\mathrm{GL}_{1}\left(\mathbb{F}_{q}\right)$, while for $q=2$, the cuspidal characters for $\mathrm{GL}_{s}\left(\mathbb{F}_{q}\right)$ with $s \geq 2$ play a role, miraculously giving the same polynomial $t_{q}(n, \ell)$ in $q$ evaluated at $q=2$.

Question 1.4 Can one derive the formulas (1.6) or (1.8) via inclusion-exclusion more directly?

Question 1.5 Can one derive Theorem 1.1 bijectively, or by an overcount, in the spirit of Dénes [6], that counts factorizations of all conjugates of a Singer cycle, and then divides by the conjugacy class size?

\section{The character theory approach to factorizations}

We recall the classical approach to factorization counts, which goes back to Frobenius [7].

Definition 2.1 Given a finite group $G$, let $\operatorname{Irr}(G)$ be the set of its irreducible ordinary (finite-dimensional, complex) representations $V$. For each $V$ in $\operatorname{Irr}(G)$, denote by $\operatorname{deg}(V)$ the degree $\operatorname{dim}_{\mathbb{C}} V$, and let $\chi_{V}(g)=\operatorname{Tr}(g: V \rightarrow V)$ be its character value at $g$, along with $\widetilde{\chi}_{V}(g):=\frac{\chi_{V}(g)}{\operatorname{deg}(V)}$ the normalized character value. Both functions $\chi_{V}(-)$ and $\widetilde{\chi}_{V}(-)$ on $G$ extend by $\mathbb{C}$-linearity to functionals on the group algebra $\mathbb{C} G$.

Proposition 2.2 (Frobenius [7]) Let $G$ be a finite group, and $A_{1}, \ldots, A_{\ell} \subset G$ unions of conjugacy classes in $G$. Then, for $g$ in $G$, the number of ordered factorizations $\left(t_{1}, \ldots, t_{\ell}\right)$ with $g=t_{1} \cdots t_{\ell}$ and $t_{i}$ in $A_{i}$ for $i=1,2, \ldots, \ell$ is

$$
\frac{1}{|G|} \sum_{V \in \operatorname{Irr}(G)} \operatorname{deg}(V) \cdot \chi_{V}\left(g^{-1}\right) \cdot \widetilde{\chi}_{V}\left(z_{1}\right) \cdots \widetilde{\chi}_{V}\left(z_{\ell}\right)
$$

where $z_{i}:=\sum_{t \in A_{i}} t$ in $\mathbb{C} G$.

This lemma was a main tool used by Jackson [15, §2], as well as by Chapuy and Stump [5, §4] in their solution of the analogous question in well-generated complex reflection groups. The proof follows from a straightforward computation in the group algebra $\mathbb{C} G$ coupled with the isomorphism of $G$-representations $\mathbb{C} G \cong \bigoplus_{V \in \operatorname{Irr}(G)} V^{\oplus \operatorname{deg}(V)}$; it may be found for example in [19, Thm. 1.1.12], [22, Thm. 2.5.9]. 


\section{Review of ordinary characters of $\mathrm{GL}_{n}\left(\mathbb{F}_{q}\right)$}

The ordinary character theory of $\mathrm{GL}_{n}:=\mathrm{GL}_{n}\left(\mathbb{F}_{q}\right)$ was worked out by Green [10], and has been reworked many times. Apart from Green's paper, some useful references for us in what follows will be Macdonald [23, Chaps. III, IV], and Zelevinsky [32, $\S 11]$.

\subsection{Parabolic or Harish-Chandra induction}

The key notion is that of parabolic or Harish-Chandra induction: given an integer composition $\alpha=\left(\alpha_{1}, \ldots, \alpha_{m}\right)$ of $n$, so that $\alpha_{i}>0$ and $|\alpha|:=\sum_{i} \alpha_{i}=n$, and class functions $f_{i}$ on $\mathrm{GL}_{\alpha_{i}}$ for $i=1,2, \ldots, m$, one produces a class function $f_{1} * f_{2} * \cdots * f_{m}$ on $\mathrm{GL}_{n}$ defined as follows. Regard the $m$-tuple $\left(f_{1}, \ldots, f_{m}\right)$ as a class function on the block upper-triangular parabolic subgroup $P_{\alpha}$ inside $\mathrm{GL}_{n}$, whose typical element is

$$
p=\left[\begin{array}{cccc}
A_{1,1} & * & \cdots & * \\
0 & A_{2,2} & \cdots & * \\
\vdots & \vdots & \ddots & \vdots \\
0 & 0 & \cdots & A_{m, m}
\end{array}\right]
$$

with $A_{i, i}$ an invertible $\alpha_{i} \times \alpha_{i}$ matrix, via $\left(f_{1}, \ldots, f_{m}\right)(p)=\prod_{i=1}^{m} f_{i}\left(A_{i, i}\right)$. Then, apply (ordinary) induction of characters from $P_{\alpha}$ up to $\mathrm{GL}_{n}$. In other words, for an element $g$ in $\mathrm{GL}_{n}$, one has

$$
\begin{aligned}
& \left(f_{1} * f_{2} * \cdots * f_{m}\right)(g):=\frac{1}{\left|P_{\alpha}\right|} \sum_{\substack{h \in G: \\
h g h^{-1} \in P_{\alpha}}} f_{1}\left(A_{1,1}\right) \cdots f_{m}\left(A_{m, m}\right) \\
& \text { if } h g h^{-1} \text { looks as in (3.1). }
\end{aligned}
$$

Identify representations $U$ up to equivalence with their characters $\chi_{U}$. The parabolic induction product $(f, g) \longmapsto f * g$ gives rise to a graded, associative product on the graded $\mathbb{C}$-vector space

$$
\mathrm{Cl}\left(\mathrm{GL}_{*}\right)=\bigoplus_{n \geq 0} \mathrm{Cl}\left(\mathrm{GL}_{n}\right)
$$

which is the direct sum of class functions on all of the general linear groups, with $\mathrm{Cl}\left(\mathrm{GL}_{0}\right)=\mathbb{C}$ by convention.

\subsection{Parametrizing the $\mathrm{GL}_{n}$-irreducibles}

A $\mathrm{GL}_{n}$-irreducible $U$ is called cuspidal if $\chi_{U}$ does not occur as a constituent in any induced character $f_{1} * f_{2}$ for compositions $n=\alpha_{1}+\alpha_{2}$ with $\alpha_{1}, \alpha_{2}>0$. Denote by $\mathrm{Cusp}_{n}$ the set of all such cuspidal irreducibles $U$ for $\mathrm{GL}_{n}$, and say that weight 
$\operatorname{wt}(U)=n$. Let $\operatorname{Par}_{n}$ denote the partitions $\lambda$ of $n$ (that is, $|\lambda|:=\sum_{i} \lambda_{i}=n$ ), and define the sets

$$
\begin{gathered}
\operatorname{Par}:=\bigsqcup_{n \geq 0} \operatorname{Par}_{n}, \\
\text { Cusp }:=\bigsqcup_{n \geq 1} \operatorname{Cusp}_{n}
\end{gathered}
$$

of all partitions, and all cuspidal representations for all groups $\mathrm{GL}_{n}$. Then, the $\mathrm{GL}_{n^{-}}$ irreducible characters can be indexed as $\operatorname{Irr}\left(\mathrm{GL}_{n}\right)=\left\{\chi^{\underline{\lambda}}\right\}$ where $\underline{\lambda}$ runs through the set of all functions

$$
\begin{aligned}
\text { Cusp } & \stackrel{\lambda}{\longrightarrow} \operatorname{Par} \\
U & \longmapsto \lambda(U)
\end{aligned}
$$

having the property that

$$
\sum_{U \in \text { Cusp }} \operatorname{wt}(U)|\lambda(U)|=n .
$$

Although Cusp is infinite, this condition (3.3) implies that $\underline{\lambda}$ can only take on finitely many non- $\varnothing$ values $\lambda\left(U_{1}\right), \ldots, \lambda\left(U_{m}\right)$, and in this case

$$
\chi^{\underline{\lambda}}=\chi^{U_{1}, \lambda\left(U_{1}\right)} * \cdots * \chi^{U_{m}, \lambda\left(U_{m}\right)}
$$

where each $\chi^{U, \lambda}$ is what Green $[10, \S 7]$ called a primary irreducible character. In particular, a cuspidal character $U$ in $\mathrm{Cusp}_{n}$ is the same as the primary irreducible $\chi^{U,(1)}$.

\subsection{Jacobi-Trudi formulas}

We recall from symmetric function theory the Jacobi-Trudi and dual Jacobi-Trudi formulas [23, I (3.4),(3.5)]. For a partition $\lambda=\left(\lambda_{1} \geq \cdots \geq \lambda_{\ell}\right)$ with largest part $m:=\lambda_{1}$, these formulas express a Schur function $s_{\lambda}$ either as an integer sum of products of complete homogeneous symmetric functions $h_{n}=s_{(n)}$, or of elementary symmetric functions $e_{n}=s_{\left(1^{n}\right)}$ :

$$
\begin{aligned}
& s_{\lambda}=\operatorname{det}\left(h_{\lambda_{i}-i+j}\right)=\sum_{w \in \mathfrak{S}_{\ell}} \operatorname{sgn}(w) h_{\lambda_{1}-1+w(1)} \cdots h_{\lambda_{\ell}-\ell+w(\ell)}, \\
& s_{\lambda}=\operatorname{det}\left(e_{\lambda_{i}^{\prime}-i+j}\right)=\sum_{w \in \mathfrak{S}_{m}} \operatorname{sgn}(w) e_{\lambda_{1}^{\prime}-1+w(1)} \cdots e_{\lambda_{m}^{\prime}-m+w(m)} .
\end{aligned}
$$

Here, $\lambda^{\prime}$ is the usual conjugate or transpose partition to $\lambda$. Also $h_{0}=e_{0}=1$ and $h_{n}=e_{n}=0$ if $n<0$.

The special primary irreducible $\mathrm{GL}_{n}$-characters $\chi^{U,(n)}, \chi^{U,\left(1^{n}\right)}$ corresponding to the single row partitions $(n)$ and single column partitions $\left(1^{n}\right)$ are called general- 
ized trivial and generalized Steinberg characters, respectively, by Silberger and Zink [27]. One has analogous formulas expressing any primary irreducible character $\chi^{U, \lambda}$ virtually in terms of parabolic induction products of such characters:

$$
\begin{aligned}
\chi^{U, \lambda} & =\sum_{w \in \mathfrak{S}_{\ell}} \operatorname{sgn}(w) \chi^{U,\left(\lambda_{1}-1+w(1)\right)} * \cdots * \chi^{U,\left(\lambda_{\ell}-\ell+w(\ell)\right)} \\
\chi^{U, \lambda} & =\sum_{w \in \mathfrak{S}_{m}} \operatorname{sgn}(w) \chi^{U,\left(1^{\lambda_{1}^{\prime}-1+w(1)}\right)} * \cdots * \chi^{U,\left(1^{\lambda_{m}^{\prime}-m+w(m)}\right)}
\end{aligned}
$$

where $\chi^{U,(n)}, \chi^{U,\left(1^{n}\right)}$ are both the zero character if $n<0$, and the trivial character $\mathbf{1}_{\mathrm{GL}_{0}}$ if $n=0$.

3.4 The cuspidal characters: indexing and notation

The set $\mathrm{Cusp}_{n}$ of cuspidal characters for $\mathrm{GL}_{n}\left(\mathbb{F}_{q}\right)$ has the same cardinality $\frac{1}{n} \sum_{d \mid n} \mu(n / d) q^{d}$ as the set of irreducible polynomials in $\mathbb{F}_{q}[x]$ of degree $n$, or the set of primitive necklaces of $n$ beads having $q$ possible colors ( $=$ free orbits under $n$-fold cyclic rotation of words in $\left.\{0,1, \ldots, q-1\}^{n}\right)$. There are at least two ways one sees $\mathrm{Cusp}_{n}$ indexed in the literature.

- Green $[10, \S 7]$ indexes $\operatorname{Cusp}_{n}$ via free orbits $[\beta]=\left\{\beta, \beta^{q}, \ldots, \beta^{q^{n-1}}\right\}$ for the action of the Frobenius map $\beta \stackrel{F}{\longmapsto} \beta^{q}$ on the multiplicative group $\mathbb{F}_{q^{n}}^{\times}$; he calls such free orbits $n$-simplices. In his notation, if $U$ lies in $\mathrm{Cusp}_{s}$ and is indexed by the orbit $[\beta]$ within $\mathbb{F}_{q^{s}}$, then the primary $\mathrm{GL}_{n}$-irreducible character $\chi^{U, \lambda}$ for a partition $\lambda$ of $\frac{n}{s}$ is (up to a sign) what he denotes $I_{s}^{\beta}[\lambda]$. The special case $I_{s}^{\beta}[(m)]$ he also denotes $I_{s}^{\beta}[m]$. Thus, the cuspidal $U$ itself is (up to sign) denoted $I_{s}^{\beta}[1]$, and he also uses the alternate terminology $J_{S}(\beta):=I_{S}^{\beta}[1]$; see $[10$, p. 433].

- Later authors index $\operatorname{Cusp}_{n}$ via free orbits $[\varphi]=\left\{\varphi, \varphi \circ F, \ldots, \varphi \circ F^{n-1}\right\}$ for the Frobenius action on the dual group $\operatorname{Hom}\left(\mathbb{F}_{q^{n}}^{\times}, \mathbb{C}^{\times}\right)$. Say that $U$ in $\operatorname{Cusp}_{n}$ is associated to the orbit $[\varphi]$ in this indexing.

When $n=1$, one simply has $\operatorname{Cusp}_{1}=\operatorname{Hom}\left(\mathbb{F}_{q}^{\times}, \mathbb{C}^{\times}\right)$. In other words, the Frobenius orbits $[\varphi]=\{\varphi\}$ are singletons, and if $U$ is associated to this orbit then $U=\varphi$ considering both as homomorphisms

$$
\mathrm{GL}_{1}\left(\mathbb{F}_{q}\right)=\mathbb{F}_{q}^{\times} \stackrel{U=\varphi}{\longrightarrow} \mathbb{C}^{\times}
$$

Although we will not need Green's full description of the characters $\chi^{U,(m)}$ and $\chi^{U, \lambda}$, we will use (in the proof of Lemma 4.8 below) the following consequence of his discussion surrounding [10, Lemma 7.2].

Proposition 3.1 For $U$ in $\mathrm{Cusp}_{s}$, every $\chi^{U,(m)}$, and hence also every primary irreducible character $\chi^{U, \lambda}$, is in the $\mathbb{Q}$-span of characters of the form $\chi_{U_{1}} * \cdots * \chi_{U_{t}}$ where $U_{i}$ is in $\operatorname{Cusp}_{n_{i}}$, with s dividing $n_{i}$ for each $i$. 


\section{Some explicit character values}

We will eventually wish to apply Proposition 2.2 with $g$ being a Singer cycle, and with the central elements $z_{i}$ being sums over classes of reflections with fixed determinants. For this one requires explicit character values on four kinds of conjugacy classes of elements in $\mathrm{GL}_{n}\left(\mathbb{F}_{q}\right)$ :

- the identity, giving the character degrees,

- the Singer cycles,

- the semisimple reflections, and

- the transvections.

We review known formulas for most of these, and derive others that we will need, in the next four subsections.

It simplifies matters that the character value $\chi^{\underline{\lambda}}\left(c^{-1}\right)$ vanishes for a Singer cycle $c$ unless $\chi^{\underline{\lambda}}=\chi^{U, \lambda}$ is a primary irreducible character and the partition $\lambda$ of $\frac{n}{s}$ takes a very special form; see Proposition 4.7 below. (This may be compared with, for example, Chapuy and Stump [5, p. 9 and Lemma 5.5].)

Definition 4.1 The hook-shaped partitions of $n$ are $\lambda=\left(n-k, 1^{k}\right)$ for $k=$ $0, \ldots, n-1$.

Thus, we only compute primary irreducible character values, sometimes only those of the form $\chi^{U,\left(\frac{n}{s}-k, 1^{k}\right)}$.

\subsection{Character values at the identity: the character degrees}

Green computed the degrees of the primary irreducible characters $\chi^{U, \lambda}$ as a product formula involving familiar quantities associated to partitions.

Definition 4.2 For a partition $\lambda$, recall $[23,(1.5)]$ the quantity $n(\lambda):=\sum_{i \geq 1}(i-1) \lambda_{i}$. For a cell $a$ in row $i$ and column $j$ of the Ferrers diagram of $\lambda$ recall the hooklength [23, Example I.1]

$$
h(a):=h_{\lambda}(a):=\lambda_{i}+\lambda_{j}^{\prime}-(i+j)+1
$$

Theorem 4.3 ([10, Theorem 12]) The primary irreducible $\mathrm{GL}_{n}$-character $\chi^{U, \lambda}$ for a cuspidal character $U$ of $\mathrm{GL}_{s}\left(\mathbb{F}_{q}\right)$ and a partition $\lambda$ of $\frac{n}{s}$ has degree

$$
\begin{aligned}
\operatorname{deg}\left(\chi^{U, \lambda}\right) & =(-1)^{n-\frac{n}{s}}(q ; q)_{n} \frac{q^{s \cdot n(\lambda)}}{\prod_{a \in \lambda}\left(1-q^{s \cdot h(a)}\right)} \\
& =(-1)^{n-\frac{n}{s}}(q ; q)_{n} s_{\lambda}\left(1, q^{s}, q^{2 s}, \ldots\right) .
\end{aligned}
$$

Here, $s_{\lambda}\left(1, q, q^{2}, \ldots\right)$ is the principal specialization $x_{i}=q^{i-1}$ of the Schur function $s_{\lambda}=s_{\lambda}\left(x_{1}, x_{2}, \ldots\right)$. Observe that this formula depends only on $\lambda$ and $s$, and not on the choice of $U \in \mathrm{Cusp}_{s}$.

Two special cases of this formula will be useful in the sequel: 
- The case of hook-shapes

$$
\operatorname{deg}\left(\chi^{U,\left(\frac{n}{s}-k, 1^{k}\right)}\right)=(-1)^{n-\frac{n}{s}} q^{s\left(\begin{array}{c}
k+1 \\
2
\end{array}\right)} \frac{(q ; q)_{n}}{\left(q^{s} ; q^{s}\right) \frac{n}{s}}\left[\begin{array}{c}
\frac{n}{s}-1 \\
k
\end{array}\right]_{q^{s}}
$$

- When $s=1$ and $U=\mathbf{1}$ is the trivial character of $\mathrm{GL}_{1}\left(\mathbb{F}_{q}\right)$, the degree is given by the usual q-hook formula [28, §7.21]

$$
\begin{aligned}
\operatorname{deg}\left(\chi^{1, \lambda}\right) & =f^{\lambda}(q):=(q ; q)_{n} \frac{q^{n(\lambda)}}{\prod_{a \in \lambda}\left(1-q^{h(a)}\right)} \\
& =(q ; q)_{n} s_{\lambda}\left(1, q, q^{2}, \ldots\right)=\sum_{Q} q^{\operatorname{maj}(Q)}
\end{aligned}
$$

where the last sum is over all standard Young tableaux $Q$ of shape $\lambda$, and maj $(Q)$ is the sum of the entries $i$ in $Q$ for which $i+1$ lies in a lower row of $Q$. (Such characters are called unipotent characters.)

\subsection{Character values on Singer cycles and regular elliptic elements}

Recall from the Introduction that a Singer cycle in $\mathrm{GL}_{n}\left(\mathbb{F}_{q}\right)$ is the image of a generator for the cyclic group $\mathbb{F}_{q^{n}}^{\times} \cong \mathbb{Z} /\left(q^{n}-1\right) \mathbb{Z}$ under any embedding $\mathbb{F}_{q^{n}}^{\times} \hookrightarrow \mathrm{GL}_{\mathbb{F}_{q}}\left(\mathbb{F}_{q^{n}}\right) \cong$ $\mathrm{GL}_{n}\left(\mathbb{F}_{q}\right)$ that comes from a choice of $\mathbb{F}_{q}$-vector space isomorphism $\mathbb{F}_{q^{n}} \cong \mathbb{F}_{q}^{n}$. (Such an embedded subgroup $\mathbb{F}_{q^{n}}^{\times}$is sometimes called a Coxeter torus or an anisotropic maximal torus.) Many irreducible $\mathrm{GL}_{n}$-character values $\chi^{\underline{\lambda}}\left(c^{-1}\right)$ vanish not only on Singer cycles, but even for a larger class of elements that we introduce in the following proposition.

Proposition 4.4 The following are equivalent for $g$ in $\mathrm{GL}_{n}\left(\mathbb{F}_{q}\right)$.

(i) No conjugates $\mathrm{hgh}^{-1}$ of $\mathrm{g}$ lie in a proper parabolic subgroup $P_{\alpha} \subsetneq \mathrm{GL}_{n}$.

(ii) There are no nonzero proper $g$-stable $\mathbb{F}_{q}$-subspaces inside $\mathbb{F}_{q}^{n}$.

(iii) The characteristic polynomial $\operatorname{det}\left(x I_{n}-g\right)$ is irreducible in $\mathbb{F}_{q}[x]$.

(iv) The element $g$ is the image of some $\beta$ in $\mathbb{F}_{q^{n}}^{\times}$satisfying $\mathbb{F}_{q}(\beta)=\mathbb{F}_{q^{n}}$ (that is, a primitive element for $\left.\mathbb{F}_{q^{n}}\right)$ under one of the embeddings $\mathbb{F}_{q^{n}}^{\times} \hookrightarrow \mathrm{GL}_{\mathbb{F}_{q}}\left(\mathbb{F}_{q^{n}}\right) \cong$ $\mathrm{GL}_{n}\left(\mathbb{F}_{q}\right)$.

The elements in $\mathrm{GL}_{n}\left(\mathbb{F}_{q}\right)$ satisfying these properties are called the regular elliptic elements.

Proof (i) is equivalent to (ii). A proper $\mathbb{F}_{q}$-subspace $U$, say with $\operatorname{dim}_{\mathbb{F}_{q}} U=d<n$, is $g$-stable if and only any $h$ in $\mathrm{GL}_{n}\left(\mathbb{F}_{q}\right)$ sending $U$ to the span of the first $d$ standard basis vectors in $\mathbb{F}_{q}^{n}$ has the property that $h g h^{-1}$ lies in a proper parabolic subgroup $P_{\alpha}$ with $\alpha_{1}=d$.

(ii) implies (iii). Argue the contrapositive: if $\operatorname{det}\left(x I_{n}-g\right)$ had a nonzero proper irreducible factor $f(x)$, then $\operatorname{ker}(f(g): V \rightarrow V)$ would be a nonzero proper $g$-stable subspace. 
(iii) implies (iv). If $f(x):=\operatorname{det}\left(x I_{n}-g\right)$ is irreducible in $\mathbb{F}_{q}[x]$, then $f(x)$ is also the minimal polynomial of $g$. Thus, $g$ has rational canonical form over $\mathbb{F}_{q}$ equal to the companion matrix for $f(x)$. This is the same as the rational canonical form for the image under one of the above embeddings of any $\beta$ in $\mathbb{F}_{q^{n}}^{\times}$having minimal polynomial $f(x)$, so that $\mathbb{F}_{q}(\beta) \cong \mathbb{F}_{q^{n}}$. Hence, $g$ is conjugate to the image of such an element $\beta$ embedded in $\mathrm{GL}_{n}\left(\mathbb{F}_{q}\right)$, and then equal to such an element, after conjugating the embedding.

(iv) implies (ii). Assume that $g$ is the image of such an element $\beta$ in $\mathbb{F}_{q^{n}}^{\times}$satisfying $\mathbb{F}_{q}(\beta)=\mathbb{F}_{q^{n}}$. Then, a $g$-stable $\mathbb{F}_{q}$-subspace $W$ of $\mathbb{F}_{q}^{n}$ would correspond to a subset of $W \subset \mathbb{F}_{q^{n}}$ stable under multiplication by $\mathbb{F}_{q}$ and by $\beta$, so also stable under multiplication by $\mathbb{F}_{q}(\beta)=\mathbb{F}_{q^{n}}$. This could only be $W=\{0\}$ or $W=\mathbb{F}_{q^{n}}$.

Part (iv) of Proposition 4.4 shows that Singer cycles $c$ in $G$ are always regular elliptic, since they correspond to elements $\gamma$ for which $\mathbb{F}_{q^{n}}^{\times}=\langle\gamma\rangle$, that is, to primitive roots in $\mathbb{F}_{q^{n}}$.

Definition 4.5 Recall that associated to the extension $\mathbb{F}_{q} \subset \mathbb{F}_{q^{n}}$ is the norm map

$$
\begin{aligned}
\mathbb{F}_{q^{n}} & \stackrel{N_{\mathbb{F}^{n} / \mathbb{F}_{q}}}{\longrightarrow} \mathbb{F}_{q} \\
\beta & \longmapsto \beta \cdot \beta^{q} \cdot \beta^{q^{2}} \cdots \beta^{q^{n-1}} .
\end{aligned}
$$

The well-known surjectivity of norm maps for finite fields [20, VII Exer. 28] is equivalent to the following.

Proposition 4.6 If $\mathbb{F}_{q^{n}}^{\times}=\langle\gamma\rangle$, then $\mathbb{F}_{q}^{\times}=\langle N(\gamma)\rangle$.

Proposition 4.7 Let $g$ be a regular elliptic element in $\mathrm{GL}_{n}\left(\mathbb{F}_{q}\right)$ associated to $\beta \in \mathbb{F}_{q^{n}}$, as in Proposition 4.4(iv).

(i) The irreducible character $\chi^{\underline{\lambda}}(g)$ vanishes unless $\chi^{\underline{\lambda}}$ is a primary irreducible character $\chi^{U, \lambda}$, for some $s$ dividing $n$ and some cuspidal character $U$ in $\mathrm{Cusp}_{s}$ and partition $\lambda$ in $\operatorname{Par}_{\frac{n}{s}}$.

(ii) Furthermore, $\chi^{U, \lambda}(g)=0$ except for hook-shaped partitions $\lambda=\left(\frac{n}{s}-k, 1^{k}\right)$.

(iii) More explicitly, if $U$ in $\mathrm{Cusp}_{s}$ is associated to $[\varphi]$ with $\varphi$ in $\operatorname{Hom}\left(\mathbb{F}_{q^{s}}^{\times}, \mathbb{C}^{\times}\right)$, then

$$
\begin{aligned}
& \chi^{U,\left(\frac{n}{s}-k, 1^{k}\right)}(g)=(-1)^{k} \chi^{U,\left(\frac{n}{s}\right)}(g) \\
& =(-1)^{\frac{n}{s}-k-1} \chi^{U,\left(1^{\frac{n}{s}}\right)}(g) \\
& =(-1)^{n-\frac{n}{s}-k} \sum_{j=0}^{s-1} \varphi\left(N_{\mathbb{F}_{q^{n}} / \mathbb{F}_{q^{s}}}\left(\beta^{q^{j}}\right)\right) \text {. }
\end{aligned}
$$

(iv) If in addition $g$ is a Singer cycle then

$$
\sum_{U} \chi^{U,\left(\frac{n}{s}-k, 1^{k}\right)}(g)= \begin{cases}(-1)^{n-\frac{n}{s}-k} \mu(s) & \text { if } q=2 \\ 0 & \text { if } q \neq 2\end{cases}
$$


where the sum is over all $U$ in $\mathrm{Cusp}_{s}$, and $\mu(s)$ is the usual number-theoretic Möbius function of $s$.

Proof The key point is Proposition 4.4(i), showing that regular elliptic elements $g$ are the elements whose conjugates $h g h^{-1}$ lie in no proper parabolic subgroup $P_{\alpha}$. Hence, the parabolic induction formula (3.2) shows that any properly induced character $f_{1} * \cdots * f_{m}$ will vanish on a regular elliptic element $g$.

Assertion (i) follows immediately, as (3.4) shows non-primary irreducibles are properly induced.

Assertion (ii) also follows, as a non-hook partition $\lambda=\left(\lambda_{1} \geq \lambda_{2} \geq \cdots\right)$ has $\lambda_{2} \geq 2$, so that in the Jacobi-Trudi-style formula (3.5) for $\chi^{U, \lambda}$, each term

$$
\chi^{U,\left(\lambda_{1}-1+w(1)\right)} * \chi^{U,\left(\lambda_{2}-2+w(2)\right)} * \cdots * \chi^{U,\left(\lambda_{\ell}-\ell+w(\ell)\right)}
$$

begins with two nontrivial product factors, so it is properly induced, and vanishes on regular elliptic $g$.

The first two equalities asserted in (iii) follow from similar analysis of terms in (3.5), (3.6) for $\chi^{U, \lambda}$ when $\lambda=\left(\frac{n}{s}-k, 1^{k}\right)$. These formulas have $2^{k+1}$ and $2^{\frac{n}{s}-k}$ nonvanishing terms, respectively, of the form

$$
\begin{array}{cl}
(-1)^{k-m} & \chi^{U,\left(\alpha_{1}\right)} * \chi^{U,\left(\alpha_{2}\right)} * \cdots * \chi^{U,\left(\alpha_{m}\right)} \\
(-1)^{\frac{n}{s}-k-m} & \chi^{U,\left(1^{\beta_{1}}\right)} * \chi^{U,\left(1^{\beta_{2}}\right)} * \cdots * \chi^{U,\left(1^{\beta_{m}}\right)}
\end{array}
$$

corresponding to compositions $\left(\alpha_{1}, \ldots, \alpha_{m}\right)$ and $\left(\beta_{1}, \ldots, \beta_{m}\right)$ of $\frac{n}{s}$ with $\alpha_{1} \geq k+1$ and $\beta_{1} \geq \frac{n}{s}-k$, respectively. All such terms vanish on regular elliptic $g$, being properly induced, except the $m=1$ terms:

$$
\begin{aligned}
\chi^{U,\left(\frac{n}{s}-k, 1^{k}\right)}(g) & =(-1)^{k-1} \chi^{U,\left(\frac{n}{s}\right)}(g) \\
& =(-1)^{\frac{n}{s}-k-1} \chi^{U,\left(1^{\frac{n}{s}}\right)}(g)
\end{aligned}
$$

The last equality in (iii) comes from a result of Silberger and Zink [27, Theorem 6.1], which they deduced by combining various formulas from Green [10].

For assertion (iv), say that the regular elliptic element $g$ corresponds to an element $\beta$ in $\mathbb{F}_{q^{n}}$ under the embedding $\mathbb{F}_{q^{n}}^{\times} \hookrightarrow \operatorname{GL}_{n}\left(\mathbb{F}_{q}\right)$, and let $\gamma:=N_{\mathbb{F}_{q^{n}} / \mathbb{F}_{q^{s}}}(\beta)$ be its norm in $\mathbb{F}_{q^{s}}^{\times}$. Assertion (iii) and the multiplicative property of the norm map $N_{\mathbb{F}_{q^{n}} / \mathbb{F}_{q^{s}}}$ imply

$$
\sum_{U} \chi^{U,\left(\frac{n}{s}-k, 1^{k}\right)}(g)=(-1)^{n-\frac{n}{s}-k} \sum_{U} \sum_{j=0}^{s-1} \varphi\left(\gamma^{q^{j}}\right)=(-1)^{n-\frac{n}{s}-k} \sum_{U} \sum_{\gamma^{\prime}} \varphi\left(\gamma^{\prime}\right)
$$

where the inner sum runs over all $\gamma^{\prime}$ lying in the Frobenius orbit of $\gamma$ within $\mathbb{F}_{q^{s}}$. When one further assumes that $g$ is a Singer cycle, then Proposition 4.6 implies $\mathbb{F}_{q^{s}}^{\times}=\langle\gamma\rangle$, so that a homomorphism $\varphi: \mathbb{F}_{q^{s}}^{\times} \rightarrow \mathbb{C}^{\times}$is completely determined by its value $z:=\varphi(\gamma)$ in $\mathbb{C}^{\times}$. Furthermore, $\varphi$ will have a free Frobenius orbit if and only 
if the powers $\left\{z, z^{q}, z^{q^{2}}, \cdots, z^{q^{s-1}}\right\}$ are distinct roots of unity. Thus, one can rewrite the rightmost summation $\sum_{U} \sum_{\gamma^{\prime}} \varphi\left(\gamma^{\prime}\right)$ in (4.3) above as the sum over all $z$ in $\mathbb{C}^{\times}$ for which $z^{q^{s}-1}=1$ but $z^{q^{t}-1} \neq 1$ for any proper divisor $t$ of $s$. Number-theoretic Möbius inversion shows this is $\sum_{t \mid s} \mu\left(\frac{s}{t}\right) f(t)$ where

$$
f(t):=\sum_{\substack{z \in \mathbb{C}^{\times}: \\ z^{q^{t}-1}=1}} z= \begin{cases}1 & \text { if } q=2, t=1, \\ 0 & \text { if } q \neq 2 \text { or } t \neq 1 .\end{cases}
$$

Hence

$$
\sum_{U} \sum_{\gamma^{\prime}} \varphi\left(\gamma^{\prime}\right)= \begin{cases}\mu(s) & \text { if } q=2 \\ 0 & \text { if } q \neq 2\end{cases}
$$

\subsection{Character values on semisimple reflections}

Recall that a semisimple reflection $t$ in $\mathrm{GL}_{n}\left(\mathbb{F}_{q}\right)$ has conjugacy class determined by its non-unit eigenvalue $\operatorname{det}(t)$, lying in $\mathbb{F}_{q}^{\times} \backslash\{1\}$. Recall also the notion of the content $c(a):=j-i$ of a cell $a$ lying in row $i$ and column $j$ of the Ferrers diagram for a partition $\lambda$.

Lemma 4.8 Let $t$ be a semisimple reflection in $\mathrm{GL}_{n}\left(\mathbb{F}_{q}\right)$.

(i) Primary irreducible characters $\chi^{U, \lambda}$ vanish on t unless $\mathrm{wt}(U)=1$, that is, unless $U$ is in Cusp $_{1}$.

(ii) For $U$ in $\mathrm{Cusp}_{1}$, so $\mathbb{F}_{q}^{\times} \stackrel{U}{\rightarrow} \mathbb{C}^{\times}$, and $\lambda$ in $\operatorname{Par}_{n}$, the normalized character $\widetilde{\chi}^{U, \lambda}$ has value on $t$

$$
\tilde{\chi}^{U, \lambda}(t)=U(\operatorname{det}(t)) \cdot \frac{\sum_{a \in \lambda} q^{c(a)}}{[n]_{q}} .
$$

(iii) In particular, for $U$ in $\mathrm{Cusp}_{1}$ and hook shapes $\lambda=\left(n-k, 1^{k}\right)$, this simplifies to

$$
\tilde{\chi}^{U,\left(n-k, 1^{k}\right)}(t)=U(\operatorname{det}(t)) \cdot q^{-k} .
$$

Proof For assertion (i), we start with the fact proven by Green [10, §5 Example (ii), p. 430] that cuspidal characters for $\mathrm{GL}_{n}$ vanish on non-primary conjugacy classes, that is, those for which the characteristic polynomial is divisible by at least two distinct irreducible polynomials in $\mathbb{F}_{q}[x]$.

This implies cuspidal characters for $\mathrm{GL}_{n}$ with $n \geq 2$ vanish on semisimple reflections $t$, since such $t$ are non-primary: $\operatorname{det}(x I-t)$ is divisible by both $x-1$ and $x-\alpha$ where $\alpha=\operatorname{det}(t) \neq 1$.

Next, the parabolic induction formula (3.2) shows that any character of the form $\chi_{U_{1}} * \cdots * \chi_{U_{\ell}}$ in which each $U_{i}$ is a $\mathrm{GL}_{n_{i}}$-cuspidal with $n_{i} \geq 2$ will also vanish on 
all semisimple reflections $t$ : whenever $h t h^{-1}$ lies in the parabolic $P_{\left(n_{1}, \ldots, n_{\ell}\right)}$ and has diagonal blocks $\left(g_{1}, \ldots, g_{\ell}\right)$, one of the $g_{i_{0}}$ is also a semisimple reflection, so that $\chi_{U_{i_{0}}}\left(g_{i_{0}}\right)=0$ by the above discussion.

Lastly, Lemma 3.1 shows that every primary irreducible $\chi^{U, \lambda}$ with $\operatorname{wt}(U) \geq 2$ will vanish on every semisimple reflection: $\chi^{U, \lambda}$ is in the $\mathbb{Q}$-span of characters $\chi_{U_{1}} * \cdots * \chi_{U_{\ell}}$ with each $U_{i}$ a GL$n_{i}$-cuspidal in which wt $(U)$ divides $n_{i}$, so that $n_{i} \geq 2$.

Assertion (iii) is an easy calculation using assertion (ii), so it only remains to prove (ii). We first claim that one can reduce to the case where character $U$ in $\operatorname{Cusp}_{1}$ is the trivial character $\mathbb{F}_{q}^{\times} \stackrel{U=1}{\longrightarrow} \mathbb{C}^{\times}$. This is because one has $\chi^{U,(n)}=U=U \otimes \chi^{1,(n)}$ and hence using (3.5) one has

$$
\chi^{U, \lambda}=U \otimes \chi^{1, \lambda} \quad \text { for } \lambda \text { in } \operatorname{Par}_{n} \text { when } U \text { lies in Cusp } 1
$$

Thus, without loss of generality, $U=\mathbf{1}$, and we wish to show

$$
\widetilde{\chi}^{\mathbf{1}, \lambda}(t)=\frac{1}{[n]_{q}} \sum_{a \in \lambda} q^{c(a)}
$$

Lemma 4.9 A semisimple reflection $t$ has $\chi^{1, \lambda}(t)=\Psi\left(s_{\lambda}\right)$ where $\Psi$ is the linear map on the symmetric functions $\Lambda=\mathbb{Q}\left[p_{1}, p_{2}, \ldots\right]$ expressed in terms of power sums that sends $f\left(x_{1}, x_{2}, \ldots\right) \mapsto(q ; q)_{n-1} \frac{\partial f}{\partial p_{1}}\left(1, q, q^{2}, \ldots\right)$.

Proof (Proof of Lemma 4.9) By linearity and (3.5), it suffices to check for compositions $\alpha=\left(\alpha_{1}, \ldots, \alpha_{m}\right)$ of $n$ that $\chi^{1, \alpha}:=\chi^{1,\left(\alpha_{1}\right)} * \cdots * \chi^{1,\left(\alpha_{m}\right)}$ has $\chi^{1, \alpha}(t)=\Psi\left(h_{\alpha}\right)$ where $h_{\alpha}=h_{\alpha_{1}} \cdots h_{\alpha_{m}}$. The character $\chi^{1, \alpha}$ is just the usual induced character $\operatorname{Ind}_{P_{\alpha}}^{\mathrm{GL}_{n}} \mathbf{1}_{P_{\alpha}}$, so the permutation character on the set of $\alpha$-flags of subspaces

$$
\{0\} \subset V_{\alpha_{1}} \subset V_{\alpha_{1}+\alpha_{2}} \subset \cdots \subset V_{\alpha_{1}+\alpha_{2}+\cdots+\alpha_{m-1}} \subset \mathbb{F}_{q}^{n},
$$

which are counted by the $q$-multinomial coefficient

$$
\left[\begin{array}{c}
n \\
\alpha
\end{array}\right]_{q}:=\left[\begin{array}{c}
n \\
\alpha_{1}, \ldots, \alpha_{m}
\end{array}\right]_{q}=\frac{[n] !_{q}}{\left[\alpha_{1}\right] !_{q} \cdots\left[\alpha_{m}\right] !_{q}}=(q ; q)_{n} h_{\alpha}\left(1, q, q^{2}, \ldots\right)
$$

Thus, $\chi^{\mathbf{1}, \alpha}(t)$ counts the number of such flags stabilized by the semisimple reflection $t$. To count these let $H$ and $L$ denote, respectively, the fixed hyperplane for $t$ and the line which is the $\operatorname{det}(t)$-eigenspace for $t$. Then, one can classify the $\alpha$-flags stabilized by $t$ according to the smallest index $i$ for which $L \subset V_{\alpha_{1}+\cdots+\alpha_{i}}$. Fixing this index $i$, such flags must have their first $i-1$ subspaces $V_{\alpha_{1}}, V_{\alpha_{1}+\alpha_{2}}, \ldots, V_{\alpha_{1}+\cdots+\alpha_{i-1}}$ lying inside $H$, and their remaining subspaces from $V_{\alpha_{1}+\cdots+\alpha_{i}}$ onward containing $L$. From this description, it is not hard to see that the quotient map $\mathbb{F}_{q}^{n} \rightarrow \mathbb{F}_{q}^{n} / L$ is a bijection between such $t$-stable $\alpha$-flags and the $\left(\alpha-e_{i}\right)$-flags in $\mathbb{F}_{q}^{n} / L \cong \mathbb{F}_{q}^{n-1}$, where $\alpha-e_{i}:=$ $\left(\alpha_{1}, \ldots, \alpha_{i-1}, \alpha_{i}-1, \alpha_{i+1}, \ldots, \alpha_{m}\right)$. Consequently 


$$
\begin{aligned}
\chi^{1, \alpha}(t) & =\sum_{i=1}^{m}\left[\begin{array}{c}
n-1 \\
\alpha-e_{i}
\end{array}\right]_{q}=(q ; q)_{n-1} \sum_{i=1}^{m} h_{\alpha-e_{i}}\left(1, q, q^{2}, \ldots\right) \\
& =(q ; q)_{n-1} \frac{\partial h_{\alpha}}{\partial p_{1}}\left(1, q, q^{2}, \ldots\right)=\Psi\left(h_{\alpha}\right)
\end{aligned}
$$

using the fact [23, Example I.5.3] that $\frac{\partial h_{n}}{\partial p_{1}}=h_{n-1}$, and hence $\frac{\partial h_{\alpha}}{\partial p_{1}}=\sum_{i=1}^{m} h_{\alpha-e_{i}}$ via the Leibniz rule.

Resuming the proof of (4.6), since [23, Example I.5.3] shows $\partial s_{\lambda} / \partial p_{1}=$ $\sum_{\mu \subset \lambda:|\mu|=|\lambda|-1} s_{\mu}$, one concludes from Lemma 4.9 and (4.2) that

$$
\begin{aligned}
\tilde{\chi}^{\mathbf{1}, \lambda}(t) & =\frac{\chi^{\mathbf{1}, \lambda}(t)}{\operatorname{deg}\left(\chi^{\mathbf{1}, \lambda}\right)}=\sum_{\substack{\mu \subset \lambda: \\
|\mu|=|\lambda|-1}} \frac{(q ; q)_{n-1} s_{\mu}\left(1, q, q^{2}, \ldots\right)}{(q ; q)_{n} s_{\lambda}\left(1, q, q^{2}, \ldots\right)} \\
& =\sum_{\substack{\mu \subset \lambda: \\
|\mu|=|\lambda|-1}} \frac{f^{\mu}(q)}{f^{\lambda}(q)}
\end{aligned}
$$

where $f^{\lambda}(q)$ is the $q$-hook formula from (4.2). Thus, the desired Eq. (4.6) becomes the assertion

$$
\sum_{\substack{\mu \subset \lambda: \\|\mu|=|\lambda|-1}} \frac{f^{\mu}(q)}{f^{\lambda}(q)}=\frac{1}{[n]_{q}} \sum_{a \in \lambda} q^{c(a)}
$$

which follows from either of two results in the literature: (4.7) is equivalent ${ }^{1}$, after sending $q \mapsto q^{-1}$, to a result of Kerov [18, Theorem 1 and Eqn. (2.2)], and (4.7) is also the $t=q^{-1}$ specialization of a result of Garsia and Haiman [8, (I.15), Theorem 2.3].

\subsection{Character values on transvections}

The $\mathrm{GL}_{n}$-irreducible character values on transvections appear in probabilistic work of M. Hildebrand [12]. For primary irreducible characters, his result is equivalent ${ }^{2}$ to the following.

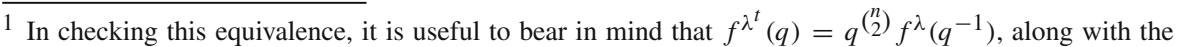
fact that if $\mu \subset \lambda$ with $|\mu|=|\lambda|-1$ and the unique cell of $\lambda / \mu$ lies in row $i$ and column $j$, then $n(\lambda)-n(\mu)=i-1$ and $n\left(\lambda^{t}\right)-n\left(\mu^{t}\right)=j-1$.

2 In seeing this equivalence, note that Hildebrand uses Macdonald's indexing [23, p. 278] of $\mathrm{GL}_{n^{-}}$ irreducibles, where partition values are transposed in the functions $\underline{\lambda}$ : Cusp $\longrightarrow$ Par relative to our convention in Sects. 3.2 and 3.3.
} 
Theorem 4.10 ([12, Theorem 2.1]) For $U$ in $\operatorname{Cusp}_{s}$ with $\lambda$ in $\operatorname{Par}_{\frac{n}{s}}$, a transvection $t$ in $\mathrm{GL}_{n}\left(\mathbb{F}_{q}\right)$ has

$$
\tilde{\chi}^{U, \lambda}(t)= \begin{cases}\frac{1}{1-q^{n-1}}\left(1-q^{n-1} \sum_{\substack{\mu \subset \lambda: \\|\mu|=|\lambda|-1}} \frac{f^{\mu}(q)}{f^{\lambda}(q)}\right) & \text { if } s=1, \\ \frac{1}{1-q^{n-1}} & \text { if } s \geq 2 .\end{cases}
$$

One can rephrase the $s=1$ case similarly to Lemma 4.8(ii).

Corollary 4.11 For $U$ in $\mathrm{Cusp}_{1}$ with $\lambda$ in $\operatorname{Par}_{n}$, a transvection t in $\mathrm{GL}_{n}\left(\mathbb{F}_{q}\right)$ has

$$
\tilde{\chi}^{U, \lambda}(t)=\frac{1-q^{n-1}\left(\frac{\sum_{a \in \lambda} q^{c(a)}}{[n]_{q}}\right)}{1-q^{n-1}} .
$$

In particular, for $U$ in $\mathrm{Cusp}_{1}$ and $0 \leq k \leq n-1$, one has

$$
\tilde{\chi}^{U,\left(n-k, 1^{k}\right)}(t)=\frac{1-q^{n-k-1}}{1-q^{n-1}}
$$

Proof The first assertion follows from Theorem 4.10 using (4.7), and the second from the calculation

$$
\sum_{a \in\left(n-k, 1^{k}\right)} q^{c(a)}=q^{-k}+q^{-k+1}+\cdots+q^{n-k-1}=q^{-k}[n]_{q} .
$$

\section{Proofs of Theorems 1.2 and 1.3.}

In proving the main results Theorems 1.2 and 1.3, it is convenient to know the equivalences between the various formulas that they assert. After checking this in Proposition 5.1, we assemble the normalized character values on reflection conjugacy class sums, in the form needed to apply (2.1). This is then used to prove Theorem 1.3 for $q>2$, from which we derive Theorem 1.2 for $q>2$. Lastly, we prove Theorem 1.2 for $q=2$. 
5.1 Equivalences of the formulas

We will frequently use the easy calculation

$$
\left[\Delta_{q}^{N}\left(x^{A}\right)\right]_{x=1}=\frac{\left(q^{A-N+1} ; q\right)_{N}}{(1-q)^{N}}
$$

which can be obtained by iterating $\Delta_{q}$, or via (1.7) and the q-binomial theorem $[9$, p. 25, Exer. 1.2(vi)]

$$
(x ; q)_{N}=\sum_{k=0}^{N}(-x)^{k} q^{\left(\begin{array}{c}
k \\
2
\end{array}\right)}\left[\begin{array}{l}
N \\
k
\end{array}\right]_{q} .
$$

The following assertion was promised in the Introduction.

Proposition 5.1 As polynomials in $q$,

(i) the three expressions (1.4), (1.5), (1.6) for $t_{q}(n, \ell)$ asserted in Theorem 1.2 all agree, and

(ii) the two expressions (1.8), (1.9) for $t_{q}(n, \ell, m)$ asserted in Theorem 1.3 agree if $m \leq \ell-1$.

Proof Assertion (i). Starting with (1.5)

$$
t_{q}(n, \ell)=(1-q)^{-1} \frac{\left(-[n]_{q}\right)^{\ell}}{[n] !_{q}}\left[\Delta_{q}^{n-1}\left(\frac{1}{x}-\frac{(1+x(1-q))^{\ell}}{x}\right)\right]_{x=1}
$$

linearity of the operator $g(x) \longmapsto\left[\Delta_{q}^{n-1} g(x)\right]_{x=1}$ lets one expand in two different ways its subexpression

$$
\left[\Delta_{q}^{n-1}\left(\frac{1}{x}-f(x)\right)\right]_{x=1} \quad \text { where } f(x):=\frac{(1+x(1-q))^{\ell}}{x} .
$$

The first way will yield (1.4), by expanding (5.3) as $\left[\Delta_{q}^{n-1}\left(\frac{1}{x}\right)\right]_{x=1}$ $-\left[\Delta_{q}^{n-1} f(x)\right]_{x=1}$. Note that

$$
\left[\Delta_{q}^{n-1}\left(\frac{1}{x}\right)\right]_{x=1}=\frac{\left(q^{1-n} ; q\right)_{n-1}}{(1-q)^{n-1}}=\frac{(-1)^{n-1}}{q^{\left(\begin{array}{l}
n \\
2
\end{array}\right)(1-q)^{n-1}}(q ; q)_{n-1}}
$$

via (5.1), which accounts for the $(-1)^{n-1}(q ; q)_{n-1}$ term inside the large parentheses of (1.4). Meanwhile, applying (1.7) to $\left[\Delta_{q}^{n-1} f(x)\right]_{x=1}$ and noting that $f\left(q^{n-1-k}\right)=$ $q^{1-n} q^{k}\left(1+q^{n-k-1}-q^{n-k}\right)^{\ell}$, one obtains a summation that accounts for the remaining terms inside the large parentheses of (1.4). This shows the equivalence of (1.4), (1.5). 
The second way will yield (1.6), by expanding $f(x)=\sum_{i=0}^{\ell}\left(\begin{array}{c}\ell \\ i\end{array}\right)(1-q)^{\ell-i} x^{\ell-i-1}$, and noting that the $i=\ell$ term cancels with the $\frac{1}{x}$ appearing inside (5.3). Therefore, (1.5) becomes

$$
\begin{aligned}
t_{q}(n, \ell) & =\left(-[n]_{q}\right)^{\ell} \frac{(1-q)^{n-1}}{(q ; q)_{n}} \sum_{i=0}^{\ell-1}-\left(\begin{array}{l}
\ell \\
i
\end{array}\right)(1-q)^{\ell-i}\left[\Delta_{q}^{n-1}\left(x^{\ell-i-1}\right)\right]_{x=1} \\
& =[n]_{q}^{\ell-1} \sum_{i=0}^{\ell-n}(-1)^{i}(q-1)^{\ell-i-1}\left(\begin{array}{l}
\ell \\
i
\end{array}\right)\left[\begin{array}{c}
\ell-i-1 \\
n-1
\end{array}\right]_{q},
\end{aligned}
$$

using (5.1). The summands with $\ell-n+1 \leq i \leq \ell-1$ vanish, showing the equivalence of (1.5), (1.6).

Assertion (ii). Starting with (1.9),

$$
t_{q}(n, \ell, m)=\frac{[n]_{q}^{\ell}}{[n] !_{q}}\left[\Delta_{q}^{n-1}\left((x-1)^{m} x^{\ell-m-1}\right)\right]_{x=1}
$$

expand the $(x-1)^{m}$ factor via the binomial theorem. Using (5.1), this expression for $t_{q}(n, \ell, m)$ becomes

$$
t_{q}(n, \ell, m)=[n]_{q}^{\ell-1} \sum_{i=0}^{m}(-1)^{i}\left(\begin{array}{c}
m \\
i
\end{array}\right)\left[\begin{array}{c}
\ell-i-1 \\
n-1
\end{array}\right]_{q} .
$$

As $i \leq m \leq \ell-1$, one has $\ell-i-1 \geq 0$ and the sum is actually over $0 \leq i \leq \ell-n$, agreeing with (1.8).

5.2 The normalized characters on reflection conjugacy class sums

Definition 5.2 For $\alpha$ in $\mathbb{F}_{q}^{\times}$, let $z_{\alpha}:=\sum_{t: \operatorname{det}(t)=\alpha} t$ in $\mathbb{C G L}_{n}$ be the sum of reflections of determinant $\alpha$.

Corollary 5.3 For $U$ in $\mathrm{Cusp}_{s}$, and $k$ in the range $0 \leq k \leq \frac{n}{s}$, and any $\alpha$ in $\mathbb{F}_{q}^{\times} \backslash\{1\}$, one has

$$
\begin{aligned}
& \tilde{\chi}^{U,\left(\frac{n}{s}-k, 1^{k}\right)}\left(z_{\alpha}\right)=[n]_{q}\left\{\begin{array}{ll}
q^{n-k-1} U(\alpha) & \text { if } s=1 \\
0 & \text { if } s \geq 2 .
\end{array}\right\}, \\
& \tilde{\chi}^{U,\left(\frac{n}{s}-k, 1^{k}\right)}\left(z_{1}\right)=[n]_{q}\left\{\begin{array}{ll}
q^{n-k-1}-1 & \text { if } s=1 \\
-1 & \text { if } s \geq 2 .
\end{array}\right\} .
\end{aligned}
$$

Proof First we count the reflections $t$ in $\mathrm{GL}_{n}\left(\mathbb{F}_{q}\right)$. There are $[n]_{q}=1+q+q^{2}+$ $\cdots+q^{n-1}$ choices for the hyperplane $H$ fixed by $t$. To count the reflections fixing $H$, without loss of generality one can conjugate $t$ and assume that $H$ is the hyperplane spanned by the first $n$ standard basis vectors $e_{1}, \ldots, e_{n-1}$. 
If $t$ is a semisimple reflection then its conjugacy class is determined by its determinant, lying in $\mathbb{F}_{q}^{\times} \backslash\{1\}$. Having fixed $\alpha:=\operatorname{det}(t)$, there will be $q^{n-1}$ such reflections that fix $e_{1}, \ldots, e_{n-1}$ : each is determined by sending $e_{n}$ to $\alpha e_{n}+\sum_{i=1}^{n-1} c_{i} e_{i}$ for some $\left(c_{1}, \ldots, c_{n-1}\right)$ in $\mathbb{F}_{q}^{n-1}$. Hence, (5.4) follows from Lemma 4.8.

The nonsemisimple reflections $t$ are the transvections, forming a single conjugacy class, with $\operatorname{det}(t)=1$. There will be $q^{n-1}-1$ transvections that fix $e_{1}, \ldots, e_{n-1}$ : each is determined by sending $e_{n}$ to $e_{n}+\sum_{i=1}^{n-1} c_{i} e_{i}$ for some $\left(c_{1}, \ldots, c_{n-1}\right)$ in $\mathbb{F}_{q}^{n-1} \backslash\{\mathbf{0}\}$. Hence (5.5) follows from Theorem 4.10 and Corollary 4.11.

\subsection{Proof of Theorem 1.3 for $q>2$.}

For a Singer cycle $c$ in $\operatorname{GL}_{n}\left(\mathbb{F}_{q}\right)$, and $\alpha=\left(\alpha_{1}, \ldots, \alpha_{\ell}\right)$ in $\left(\mathbb{F}_{q}^{\times}\right)^{\ell}$ with $\prod_{i=1}^{\ell} \alpha_{i}=$ $\operatorname{det}(c)$, Proposition 2.2 counts the reflection factorizations $c=t_{1} t_{2} \cdots t_{\ell}$ with $\operatorname{det}\left(t_{i}\right)=$ $\alpha_{i}$ as

$$
\frac{1}{\left|\mathrm{GL}_{n}\right|} \sum_{\substack{s, U): \\ s \mid n \\ U \in \mathrm{Cusp}_{s}}} \sum_{k=0}^{\frac{n}{s}-1} \operatorname{deg}\left(\chi^{U,\left(\frac{n}{s}-k, 1^{k}\right)}\right) \cdot \chi^{U,\left(\frac{n}{s}-k, 1^{k}\right)}\left(c^{-1}\right) \cdot \prod_{i=1}^{\ell} \tilde{\chi}^{U,\left(\frac{n}{s}-k, 1^{k}\right)}\left(z_{\alpha_{i}}\right) .
$$

There are several simplifications in this formula.

Firstly, note that the outermost sum over pairs $(s, U)$ reduces to the pairs with $s=1$ : since $\operatorname{det}(c)$ is a primitive root in $\mathbb{F}_{q}^{\times}$by Proposition 4.6 and $q>2$, one knows that $\operatorname{det}(c) \neq 1$, so that at least one of the $\alpha_{i}$ is not 1 . Thus, its factor $\widetilde{\chi}^{U,\left(\frac{n}{s}-k, 1^{k}\right)}\left(z_{\alpha_{i}}\right)$ in the last product will vanish if $s \geq 2$ by (5.4).

Secondly, when $s=1$ then Corollary 5.3 evaluates the product in (5.6) as

$$
\prod_{i=1}^{\ell} \tilde{\chi}^{U,\left(\frac{n}{s}-k, 1^{k}\right)}\left(z_{\alpha_{i}}\right)=[n]_{q}^{\ell}\left(q^{n-k-1}-1\right)^{m} q^{(n-k-1)(\ell-m)} U(\operatorname{det}(c))
$$

if exactly $m$ of the $\alpha_{i}$ are equal to 1 , that is, if the number of transvections in the factorization is $m$. This justifies calling it $t_{q}(n, \ell, m)$ where $m \leq \ell-1$.

Thirdly, for $s=1$ Proposition 4.7(iii) shows $^{3}$ that $\chi^{U,\left(n-k, 1^{k}\right)}\left(c^{-1}\right)=$ $(-1)^{k} U\left(\operatorname{det}\left(c^{-1}\right)\right)$, so there will be cancellation of the factor $U(\operatorname{det}(c))$ occurring in (5.7) within each summand of (5.6).

Thus, plugging in the degree formula from the $s=1$ case of (4.1), one obtains the following formula for (5.6), which we denote by $t_{q}(n, \ell, m)$, emphasizing its dependence only on $\ell$ and $m$, not on the sequence $\alpha$ :

\footnotetext{
${ }_{3}$ Here, we use the fact that $c^{-1}$ is also a Singer cycle.
} 
$t_{q}(n, \ell, m)=\frac{(q-1)[n]_{q}^{\ell}}{\left|\mathrm{GL}_{n}\right|} \sum_{k=0}^{n-1} q^{\left(\begin{array}{c}k+1 \\ 2\end{array}\right)}\left[\begin{array}{c}n-1 \\ k\end{array}\right]_{q}(-1)^{k}\left(q^{n-k-1}-1\right)^{m} q^{(n-k-1)(\ell-m)}$.

This expression may be rewritten using the $q$-difference operator $\Delta_{q}$ and (1.7) as

$$
t_{q}(n, \ell, m)=\frac{[n]_{q}^{\ell}}{\left|\mathrm{GL}_{n}\right|} q^{\left(\begin{array}{c}
n \\
2
\end{array}\right)}(q-1)^{n}\left[\Delta_{q}^{n-1}\left((x-1)^{m} x^{\ell-m-1}\right)\right]_{x=1} .
$$

Since $\left|\mathrm{GL}_{n}\right|=q^{\left(\begin{array}{c}n \\ 2\end{array}\right)}(-1)^{n}(q ; q)_{n}$, this last expression is the same as (1.9). Hence, by Proposition 5.1, this completes the proof of Theorem 1.3 for $q>2$.

\subsection{Proof of Theorem 1.2 when $q>2$.}

We will use Theorem 1.3 for $q>2$ to derive (1.5) for $q>2$. First note that one can choose a sequence of determinants $\alpha=\left(\alpha_{1}, \ldots, \alpha_{\ell}\right)$ in $\mathbb{F}_{q}^{\times}$that has $\prod_{i=1}^{\ell} \alpha_{i}=\operatorname{det}(c)$ and has exactly $m$ of the $\alpha_{i}=1$ in a two-step process: first choose $m$ positions out of $\ell$ to have $\alpha_{i}=1$, then choose the remaining sequence in $\left(\mathbb{F}_{q}^{\times} \backslash\{1\}\right)^{\ell-m}$ with product equal to $\operatorname{det}(c)$. Simple counting shows that in a finite group $K$, the number of sequences in $(K \backslash\{1\})^{N}$ whose product is some fixed nonidentity element ${ }^{4}$ of $K$ is

$$
\frac{(|K|-1)^{N}-(-1)^{N}}{|K|}
$$

Applying this to $K=\mathbb{F}_{q}^{\times}$with $N=\ell-m$ gives

$$
t_{q}(n, \ell)=\sum_{m=0}^{\ell} t_{q}(n, \ell, m)\left(\begin{array}{c}
\ell \\
m
\end{array}\right) \frac{(q-2)^{\ell-m}-(-1)^{\ell-m}}{q-1} .
$$

Thus, from (1.9) one has

$$
\begin{aligned}
t_{q}(n, \ell) Z= & \frac{(q-1)[n]_{q}^{\ell}}{\left|\mathrm{GL}_{n}\right|} q^{\left(\begin{array}{c}
n \\
2
\end{array}\right)}(q-1)^{n-1} \\
& \times\left[\Delta_{q}^{n-1}\left(\sum_{m=0}^{\ell}\left(\begin{array}{l}
\ell \\
m
\end{array}\right)(x-1)^{m} x^{\ell-m-1} \frac{(q-2)^{\ell-m}-(-1)^{\ell-m}}{q-1}\right)\right]_{x=1}
\end{aligned}
$$

\footnotetext{
${ }^{4}$ In fact, Theorem 1.2 is stated for $n \geq 2$, but remains valid for when $n=1$ and $q>2$. It is only in the trivial case where $\mathrm{GL}_{1}\left(\mathbb{F}_{2}\right)=\{1\}$ that the "Singer cycle" $c$ is actually the identity element, so that the count (5.8) fails.
} 


$$
\begin{aligned}
& =\frac{\left(-[n]_{q}\right)^{\ell}}{\left|\mathrm{GL}_{n}\right|} q^{\left(\begin{array}{c}
n \\
2
\end{array}\right)}(q-1)^{n-1}\left[\Delta_{q}^{n-1}\left(\frac{(1+x(1-q))^{\ell}}{x}-\frac{1}{x}\right)\right]_{x=1} \\
& =(1-q)^{-1} \frac{\left(-[n]_{q}\right)^{\ell}}{[n] !_{q}}\left[\Delta_{q}^{n-1}\left(\frac{1}{x}-\frac{(1+x(1-q))^{\ell}}{x}\right)\right]_{x=1}
\end{aligned}
$$

which is (1.5). Hence, by Proposition 5.1, this completes the proof of Theorem 1.2 when $q>2$.

5.5 Proof of Theorem 1.2 when $q=2$.

Here, all reflections are transvections and (2.1) gives us

$$
\begin{aligned}
t_{q}(n, \ell) & =\frac{1}{\left|\mathrm{GL}_{n}\right|} \sum_{\chi^{\underline{\lambda} \in \operatorname{Irr}\left(\mathrm{GL}_{n}\right)}} \operatorname{deg}\left(\chi^{\underline{\lambda}}\right) \cdot \chi^{\underline{\lambda}}\left(c^{-1}\right) \cdot \tilde{\chi}^{\underline{\lambda}}\left(z_{1}\right)^{\ell} \\
& =\frac{1}{\mid\left[\mathrm{GL}_{n} \mid\right.} \sum_{\substack{(s, U): \\
s \mid n \\
U \in \operatorname{Cusp}_{s}}}^{\sum_{\text {Call this } f(s, U)}^{\frac{n}{s}-1} \operatorname{deg}\left(\chi^{U,\left(\frac{n}{s}-k, 1^{k}\right)}\right) \cdot \chi^{U,\left(\frac{n}{s}-k, 1^{k}\right)}\left(c^{-1}\right) \cdot \tilde{\chi}^{U,\left(\frac{n}{s}-k, 1^{k}\right)}\left(z_{1}\right)^{\ell}}
\end{aligned}
$$

using the vanishing of $\chi^{\underline{\lambda}}\left(c^{-1}\right)$ from Proposition 4.7(i,ii). We separate the computation into $s=1$ and $s \geq 2$, and first compute $\sum_{U \in \mathrm{Cusp}_{1}} f(s, U)$. As $q=2$ there is only one $U$ in $\mathrm{Cusp}_{1}$, namely $U=\mathbf{1}$, and hence

$$
\begin{aligned}
\sum_{U \in \operatorname{Cusp}_{1}} f(s, U)=f(1, \mathbf{1}) & =\sum_{k=0}^{n-1} \operatorname{deg}\left(\chi^{\mathbf{1},\left(n-k, 1^{k}\right)}\right) \cdot \chi^{\mathbf{1},\left(n-k, 1^{k}\right)}\left(c^{-1}\right) \cdot \tilde{\chi}^{\mathbf{1},\left(n-k, 1^{k}\right)}(z)^{\ell} \\
& =\sum_{k=0}^{n-1} q^{\left(\begin{array}{c}
k+1 \\
2
\end{array}\right)}\left[\begin{array}{c}
n-1 \\
k
\end{array}\right]_{q} \cdot(-1)^{k} \cdot[n]_{q}^{\ell}\left(q^{n-k}-q^{n-k-1}-1\right)^{\ell}
\end{aligned}
$$

using the degree formula (4.1) at $s=1$, the fact that $\chi^{\left(1, n-k, 1^{k}\right)}\left(c^{-1}\right)=$ $(-1)^{k} \chi^{\mathbf{1},(n)}\left(c^{-1}\right)=(-1)^{k}$ from Proposition 4.7(iii), and the value $\widetilde{\chi}^{\mathbf{1},\left(n-k, 1^{k}\right)}\left(z_{1}\right)=$ $[n]_{q}\left(q^{n-k}-q^{n-k-1}-1\right)$ from $(5.5)$.

For $s \geq 2$, we compute

$$
\begin{aligned}
& \sum_{\substack{(s, U): \\
s \mid n, s \geq 2 \\
U \in \operatorname{Cusp}_{s}}} f(s, U)=\sum_{\substack{(s, U): \\
s \mid n, s \geq 2 \\
U \in \operatorname{Cusp}_{s}}} \sum_{k=0}^{\frac{n}{s}-1} \operatorname{deg}\left(\chi^{U,\left(\frac{n}{s}-k, 1^{k}\right)}\right) \cdot \chi^{U,\left(\frac{n}{s}-k, 1^{k}\right)}\left(c^{-1}\right) \\
& \cdot \tilde{\chi}^{U,\left(\frac{n}{s}-k, 1^{k}\right)}\left(z_{1}\right)^{\ell}
\end{aligned}
$$




$$
\begin{aligned}
= & \sum_{s \mid n} \sum_{\substack{s \geq 2 \\
s \geq 0}}^{\frac{n}{s}-1} \frac{(-1)^{n-\frac{n}{s}} q^{s\left(\begin{array}{c}
k+1 \\
2
\end{array}\right)}(q ; q)_{n}}{\left(q^{s} ; q^{s}\right) \frac{n}{s}}\left[\begin{array}{c}
\frac{n}{s}-1 \\
k
\end{array}\right]_{q^{s}} \\
& \cdot\left(\sum_{U \in \mathrm{Cusp}_{s}} \chi^{U,\left(\frac{n}{s}-k, 1^{k}\right)}\left(c^{-1}\right)\right) \cdot\left(-[n]_{q}\right)^{\ell}
\end{aligned}
$$

again via (4.1), Proposition 4.7(iii), and (5.5). The parenthesized sum is $(-1)^{n-\frac{n}{s}-k}$ $\mu(s)$ by Proposition 4.7(iii, iv), so

$$
\begin{aligned}
\sum_{\substack{(s, U): \\
s \mid n, s \geq 2 \\
U \in \mathrm{Cusp}_{s}}} f(s, U)= & \left(-[n]_{q}\right)^{\ell}(q ; q)_{n} \sum_{\substack{s \mid n \\
s \geq 2}} \frac{1}{\left(q^{s} ; q^{s}\right) \frac{n}{s}} \\
& \times\left(\sum_{k=0}^{\frac{n}{s}-1}(-1)^{k} q^{s\left(\begin{array}{c}
k+1 \\
2
\end{array}\right)}\left[\begin{array}{c}
\frac{n}{s}-1 \\
k
\end{array}\right]_{q^{s}}\right) \mu(s) \\
= & \left(-[n]_{q}\right)^{\ell}(q ; q)_{n} \sum_{s \mid n} \frac{\mu(s)}{\left(q^{s} ; q^{s}\right) \frac{n}{s}}\left(q^{s} ; q^{s}\right)_{\frac{n}{s}-1} \\
= & \left(-[n]_{q}\right)^{\ell}(q ; q)_{n-1} \sum_{s \mid n} \mu(s) \\
= & -\left(-[n]_{q}\right)^{\ell}(q ; q)_{n-1},
\end{aligned}
$$

where the second equality used the $q$-binomial theorem (5.2). Thus, one has for $q=2$ that

$$
\begin{aligned}
& t_{q}(n, \ell)=\frac{1}{\left|\mathrm{GL}_{n}\right|}\left(-\left(-[n]_{q}\right)^{\ell}(q ; q)_{n-1}\right. \\
& \left.+\sum_{k=0}^{n-1} q^{\left(\begin{array}{c}
k+1 \\
2
\end{array}\right)}\left[\begin{array}{c}
n-1 \\
k
\end{array}\right]_{q} \cdot(-1)^{k} \cdot[n]_{q}^{\ell}\left(q^{n-k}-q^{n-k-1}-1\right)^{\ell}\right) .
\end{aligned}
$$

Since $\left|\mathrm{GL}_{n}\right|=(-1)^{n} q^{\left(\begin{array}{c}n \\ 2\end{array}\right)}(q ; q)_{n}$, one finds that (5.9) agrees with the expression (1.4)

$$
\begin{aligned}
t_{q}(n, \ell)= & \frac{\left(-[n]_{q}\right)^{\ell}}{q^{\left(\begin{array}{c}
n \\
2
\end{array}\right)(q ; q)_{n}}}\left((-1)^{n-1}(q ; q)_{n-1}+\sum_{k=0}^{n-1}(-1)^{k+n} q^{\left(\begin{array}{c}
k+1 \\
2
\end{array}\right)}\left[\begin{array}{c}
n-1 \\
k
\end{array}\right]_{q}\right. \\
& \left.\left(1+q^{n-k-1}-q^{n-k}\right)^{\ell}\right)
\end{aligned}
$$

after redistributing the $[n]_{q}^{\ell}$ and powers of -1 . This completes the proof of Theorem 1.2 for $q=2$. 


\section{Further remarks and questions}

\subsection{Product formula versus partial fraction expansions}

The equivalence between (1.1), (1.2), and between (1.3), (1.4) are explained as follows. One checks the partial fraction expansion of (1.1) is

$$
\sum_{\ell \geq 0} t(n, \ell) x^{\ell}=\frac{n^{n-2} x^{n-1}}{\prod_{k=0}^{n-1}\left(1-x n\left(\frac{n-1}{2}-k\right)\right)}=\frac{1}{n !} \sum_{k=0}^{n-1} \frac{(-1)^{k}\left(\begin{array}{c}
n-1 \\
k
\end{array}\right)}{1-x n\left(\frac{n-1}{2}-k\right)}
$$

and comparing coefficients of $x^{\ell}$ gives the first equality in (1.2).

Similarly, one checks that the partial fraction expansion of the right side of (1.3) is

$$
\begin{aligned}
& \left(q^{n}-1\right)^{n-1} \cdot \frac{x^{n}}{\left(1+x[n]_{q}\right) \prod_{k=0}^{n-1}\left(1+x[n]_{q}\left(1+q^{k}-q^{k+1}\right)\right)} \\
& =\frac{(-1)^{n}}{q^{\left(\begin{array}{c}
n \\
2
\end{array}\right)\left(q^{n}-1\right)}}\left(\frac{1}{1+x[n]_{q}}+\sum_{k=0}^{n-1} \frac{(-1)^{k+1} q^{\left(\begin{array}{c}
k+1 \\
2
\end{array}\right)}}{(q ; q)_{k}(q ; q)_{n-1-k}}\right. \\
& \left.\cdot \frac{1}{1+x[n]_{q}\left(1+q^{n-k-1}-q^{n-k}\right)}\right) .
\end{aligned}
$$

Comparing coefficients of $x^{\ell}$ in (6.1) gives (1.4). This proves (1.3).

6.2 More observations about $t_{q}(n, \ell, m)$

From (1.6) and (1.8) one can derive $q=1$ limits

$$
\begin{aligned}
t_{1}(n, \ell) & :=\lim _{q \rightarrow 1} \frac{t_{q}(n, \ell)}{(1-q)^{n-1}}=(-n)^{\ell-1}\left(\begin{array}{c}
\ell \\
n
\end{array}\right) \\
t_{1}(n, \ell, m) & :=\lim _{q \rightarrow 1} t_{q}(n, \ell, m)=n^{\ell-1}\left(\begin{array}{c}
\ell-m-1 \\
\ell-n
\end{array}\right) .
\end{aligned}
$$

We do not know an interpretation for these limits.

\subsection{Exponential generating function}

The classical count $t(n, \ell)$ of factorizations of an $n$-cycle into $\ell$ transpositions has both an elegant ordinary generating function (1.1) and exponential generating function

$$
\sum_{\ell \geq 0} t(n, \ell) \frac{u^{\ell}}{\ell !}=\frac{1}{n !}\left(e^{u \frac{n}{2}}-e^{-u \frac{n}{2}}\right)^{n-1}
$$

This was generalized by Chapuy and Stump [5] to well-generated finite complex reflection group $W$ as follows; we refer to their paper for the background on such 
groups. If $W$ acts irreducibly on $\mathbb{C}^{n}$, with a total of $\mathrm{N}^{\text {ref }}$ reflections and $\mathrm{N}^{\text {hyp }}$ reflecting hyperplanes, then for any Coxeter element $c$, the number $a_{\ell}$ of ordered factorizations $c=t_{1} \cdots t_{\ell}$ into reflections satisfies

$$
\begin{aligned}
\sum_{\ell \geq 0} a_{\ell} \frac{u^{\ell}}{\ell !} & =\frac{1}{|W|}\left(e^{u \frac{\mathrm{N}^{\mathrm{ref}}}{n}}-e^{-u \frac{\mathrm{N} \text { hyp }}{n}}\right)^{n} \\
& =\frac{1}{|W|} e^{-u \mathrm{~N}^{\text {hyp }}}\left(e^{u \frac{\mathrm{N}^{\mathrm{ref}}+\mathrm{N}_{\mathrm{hyp}}}{n}}-1\right)^{n} \\
& =\frac{1}{|W|} e^{-u \mathrm{~N}^{\mathrm{hyp}}}\left[\Delta^{n}\left(e^{u x \frac{\mathrm{N}^{\mathrm{ref}}+\mathrm{N}^{\mathrm{hyp}}}{n}}\right)\right]_{x=0}
\end{aligned}
$$

where the last equality uses the fact that the difference operator $\Delta$ satisfies $\left[\Delta^{n}\left(e^{a x}\right)\right]_{x=0}=\left(e^{a}-1\right)^{n}$.

One can derive an exponential generating function analogous to (6.3) for the number $t_{q}(n, \ell)$ of Singer cycle factorizations in $W=\mathrm{GL}_{n}\left(\mathbb{F}_{q}\right)$,

$$
\sum_{\ell \geq 0} t_{q}(n, \ell) \frac{u^{\ell}}{\ell !}=\frac{(q-1)^{n-1} q^{\left(\begin{array}{c}
n \\
2
\end{array}\right)}}{|W|} e^{-u \mathrm{~N}^{\mathrm{hyp}}}\left[\Delta_{q}^{n-1}\left(\frac{1}{x}\left(e^{u x \frac{\mathrm{N}^{\mathrm{ref}}+\mathrm{N}^{\mathrm{hyp}}}{q^{n-1}}}-1\right)\right)\right]_{x=1}
$$

where $\mathrm{N}^{\text {hyp }}, \mathrm{N}^{\text {ref }}$ denote the number of reflecting hyperplanes and reflections in $W=$ $\mathrm{GL}_{n}\left(\mathbb{F}_{q}\right)$, that is,

$$
\begin{aligned}
\mathrm{N}^{\text {hyp }} & =[n]_{q}, \\
\mathrm{~N}^{\mathrm{ref}} & =[n]_{q}\left(q^{n}-q^{n-1}-1\right) .
\end{aligned}
$$

To prove (6.4), use (1.5) to find

$$
\begin{aligned}
& \sum_{\ell \geq 0} t_{q}(n, \ell) \frac{u^{\ell}}{\ell !}=\frac{(1-q)^{n-1}}{(q ; q)_{n}}\left[\Delta_{q}^{n-1}\left(\frac{1}{x}\left(e^{-u[n]_{q}}-e^{-u[n]_{q}(1+x(1-q))}\right)\right)\right]_{x=1} \\
& =\frac{(-1)^{n}(1-q)^{n-1} q^{\left(\begin{array}{c}
n \\
2
\end{array}\right)}}{|W|} e^{-u[n]_{q}}\left[\Delta_{q}^{n-1}\left(\frac{1}{x}\left(1-e^{\left.u x[n]_{q}(q-1)\right)}\right)\right)\right]_{x=1} .
\end{aligned}
$$

Noting that $[n]_{q}=\mathrm{N}^{\text {hyp }}$, and $[n]_{q}(q-1)=q^{n}-1=\left(\mathrm{N}^{\text {hyp }}+\mathrm{N}^{\text {ref }}\right) / q^{n-1}$, and distributing some negative signs, gives (6.4).

\subsection{Hurwitz orbits}

In a different direction, one can consider the Hurwitz action of the braid group on $\ell$ strands acting on length $\ell$ ordered factorizations $c=t_{1} t_{2} \cdots t_{\ell}$. Here, the braid group generator $\sigma_{i}$ acts on ordered factorizations as follows: 


$$
\begin{aligned}
& \left(t_{1}, \ldots, t_{i-1}, \quad t_{i}, \quad t_{i+1}, \quad t_{i+2}, \ldots, t_{\ell}\right) \stackrel{\sigma_{i}}{\longmapsto} \\
& \left(t_{1}, \ldots, t_{i-1}, t_{i+1}, t_{i+1}^{-1} t_{i} t_{i+1}, t_{i+2}, \ldots, t_{\ell}\right) .
\end{aligned}
$$

For well-generated complex reflection groups $W$ of rank $n$ and taking $\ell=n$, Bessis showed [3, Prop. 7.5] that the set of all shortest ordered factorizations $\left(t_{1}, \ldots, t_{n}\right)$ of a Coxeter element $c=t_{1} t_{2} \cdots t_{n}$ forms a single transitive orbit for this Hurwitz action.

One obvious obstruction to an analogous transitivity assertion for $c$ a Singer cycle in $\mathrm{GL}_{n}\left(\mathbb{F}_{q}\right)$ and factorizations $c=t_{1} t_{2} \cdots t_{\ell}$ is that the unordered $\ell$-element multiset $\left\{\operatorname{det}\left(t_{i}\right)\right\}_{i=1}^{\ell}$ of $\mathbb{F}_{q}^{\times}$is constant on a Hurwitz orbit, but (when $q \neq 2$ ) can vary between different factorizations, even when $\ell=n$. Nevertheless, we make the following conjecture.

Conjecture 6.1 Any two factorizations $c=t_{1} t_{2} \cdots t_{\ell}$ with the same multiset $\left\{\operatorname{det}\left(t_{i}\right)\right\}_{i=1}^{\ell}$ lie in the same Hurwitz orbit. In particular, there is only one Hurwitz orbit of factorizations when $q=2$ for any $\ell$.

We report here some partial evidence for Conjecture 6.1.

- It is true when $n=\ell=2$; here is a proof. Fix a Singer cycle $c$ in $\mathrm{GL}_{2}\left(\mathbb{F}_{q}\right)$ and $\alpha_{1}, \alpha_{2}$ in $\mathbb{F}_{q}^{\times}$having $\operatorname{det}(c)=\alpha_{1} \alpha_{2}$. Theorem 1.3 in the case $\ell=n=2$ tells us that there will be exactly [2] $]_{q}=q+1$ factorizations $c=t_{1} \cdot t_{2}$ of $c$ as a product of two reflections with $\left(\operatorname{det}\left(t_{1}\right), \operatorname{det}\left(t_{2}\right)\right)=\left(\alpha_{1}, \alpha_{2}\right)$, and similarly $q+1$ for which $\left(\operatorname{det}\left(t_{1}\right), \operatorname{det}\left(t_{2}\right)\right)=\left(\alpha_{2}, \alpha_{1}\right)$. This gives a total of either $q+1$ or $2(q+1)$ factorizations with this multiset of determinants, depending upon whether or not $\alpha_{1}=\alpha_{2}$. Now note that applying the Hurwitz action $\sigma_{1}$ twice sends

$$
\left(t_{1}, t_{2}\right) \stackrel{\sigma_{1}}{\longmapsto}\left(t_{2}, t_{2}^{-1} t_{1} t_{2}\right) \stackrel{\sigma_{1}}{\longmapsto}(t_{2}^{-1} t_{1} t_{2}, \underbrace{t_{2}^{-1} t_{1}^{-1} t_{2} t_{1} t_{2}}_{=c^{-1} t_{2} c}),
$$

yielding a factorization with the same determinant sequence, but whose second factor changes from $t_{2}$ to $c^{-1} t_{2} c$. This moves the reflecting hyperplane (line) $H$ for $t_{2}$ to the line $c^{-1} H$ for $c^{-1} t_{2} c$. Since $\mathbb{F}_{q^{2}}^{\times}=\langle c\rangle$, one knows that the powers of $c$ act transitively on the lines in $\mathbb{F}_{q^{2}} \cong \mathbb{F}_{q}^{2}$, and hence there will be at least $q+1$ different second factors $\left\{c^{-i} t_{2} c^{i}\right\}$ achieved in the Hurwitz orbit. This shows that the Hurwitz orbit contains at least $q+1$ or $2(q+1)$ different factorizations, depending upon whether or not $\alpha_{1}=\alpha_{2}$, so it exhausts the factorizations that achieve this multiset of determinants. This completes the proof.

- Conjecture 6.1 has also been checked

- for $q=2$ when $n=\ell \leq 5$ and $n=3, \ell=4$,

- for $q=3$ when $n=2$ and $\ell \leq 4$, and also when $n=\ell=3$,

- for $q=5$ when $n=2$ and $\ell \leq 3$.

One might hope to prove Conjecture 6.1 by a method similar to the uniform proof for transitivity of the Hurwitz action on short reflection factorizations of Coxeter elements in real reflection groups, given in earlier work of Bessis [2, Prop. 1.6.1]. His proof is via induction on the rank, and relies crucially on proving these facts: 
- The elements $w \leq c$ in the absolute order, that is, the elements which appear as partial products $w=t_{1} t_{2} \cdots t_{i}$ in shortest factorizations $c=t_{1} t_{2} \cdots t_{n}$, are all themselves parabolic Coxeter elements, that is, Coxeter elements for conjugates of standard parabolic subgroups of $W$.

- All such parabolic Coxeter elements share the property that the Hurwitz action is transitive on their shortest factorizations into reflections.

One encounters difficulties in trying to prove this analogously, when one examines the interval $[e, c]$ of elements lying below a Singer cycle $c$ in $\mathrm{GL}_{n}\left(\mathbb{F}_{q}\right)$ :

- It is no longer true that the elements $g$ in $[e, c]$ all have a transitive Hurwitz action on their own short factorizations. For example in $\mathrm{GL}_{4}\left(\mathbb{F}_{2}\right)$, the unipotent element $u$ equal to a single Jordan block of size 4 appears as a partial product on the way to factoring a Singer cycle, but its 64 short factorizations $u=t_{1} t_{2} t_{3}$ into reflections break up into two Hurwitz orbits, of sizes 16 and 48.

- It also seems nontrivial to characterize intrinsically the elements in $[e, c]$ for a fixed Singer cycle $c$. For example, the elements $g$ which are $c$-noncrossing in the following sense appear ${ }^{5}$ to be always among them: arranging the elements $\mathbb{F}_{q^{n}}^{\times}=$ $\left\{1, c, c^{2}, \ldots, c^{q^{n}-2}\right\}$ clockwise circularly, $g$ permutes them (after embedding them via $\mathbb{F}_{q^{n}} \cong \mathbb{F}_{q}^{n}$ ) in cycles that are each oriented clockwise, and these oriented arcs do not cross each other. However, starting already with $\mathrm{GL}_{4}\left(\mathbb{F}_{2}\right)$ and $\mathrm{GL}_{3}\left(\mathbb{F}_{3}\right)$, there are other element below the Singer cycle besides these $c$-noncrossings.

\section{$6.5 q$-Noncrossings?}

The poset of elements $[e, c]$ lying below a Singer cycle $c$ in the absolute order on $\mathrm{GL}_{n}\left(\mathbb{F}_{q}\right)$ would seem like a reasonable candidate for a $q$-analog of the usual poset of noncrossing partitions of $\{1,2, \ldots, n\}$; see [1]. However, $[e, c]$ does not seem to be so well behaved in $\mathrm{GL}_{n}\left(\mathbb{F}_{q}\right)$, although a few things were proven about it by Jia Huang in [13].

For instance, he showed that the absolute length of an element $g$ in $\mathrm{GL}_{n}\left(\mathbb{F}_{q}\right)$, that is, the minimum length of a factorization into reflections, coincides with the codimension of the fixed space $\left(\mathbb{F}_{q}^{n}\right)^{g}$. Hence, the poset $[e, c]$ is ranked in a similar fashion to the noncrossing partitions of real reflection groups, and has an order- and rank-preserving map

$$
\begin{aligned}
{[e, c] } & \stackrel{\pi}{\longrightarrow} L\left(\mathbb{F}_{q}^{n}\right) \\
g & \longmapsto \\
& \left(\mathbb{F}_{q}^{n}\right)^{g}
\end{aligned}
$$

to the lattice $L\left(\mathbb{F}_{q}^{n}\right)$ of subspaces of $\mathbb{F}_{q}^{n}$. Because conjugation by $c$ acts transitively on lines and hyperplanes, this map is surjective for $n \leq 3$; empirically, it seems to be surjective in general. The poset $[e, c]$ also has a Kreweras complementation antiautomorphism $w \mapsto w^{-1} c$.

\footnotetext{
5 That is, it is true for $\mathrm{GL}_{n}\left(\mathbb{F}_{2}\right)$ with $n=2,3,4$ and also for $\mathrm{GL}_{n}\left(\mathbb{F}_{3}\right)$ with $n=2,3$.
} 
However, Huang noted that the rank sizes of $[e, c]$ do not seem so suggestive. E.g., for $[e, c]$ in $\mathrm{GL}_{4}\left(\mathbb{F}_{2}\right)$ they are $(1,60,240,60,1)$, and preclude $\pi$ being an $N$-to-one map for some integer $N$, since $L\left(\mathbb{F}_{2}^{4}\right)$ has rank sizes $(1,15,35,15,1)$ and 35 does not divide 240.

Question 6.2 Are the c-noncrossing elements mentioned in Sect. 6.4 a better-behaved subposet of $[e, c]$ ?

\subsection{Regular elliptic elements versus Singer cycles}

Empirical evidence supports the following hypothesis regarding the regular elliptic elements of $\mathrm{GL}_{n}\left(\mathbb{F}_{q}\right)$ that appeared in Proposition 4.4.

Conjecture 6.3 The number of ordered reflection factorizations $g=t_{1} t_{2} \cdots t_{\ell}$ is the same for all regular elliptic elements $g$ in $\mathrm{GL}_{n}\left(\mathbb{F}_{q}\right)$, namely the quantity $t_{q}(n, \ell)$ that appears in Theorem 1.2.

Conjecture 6.3 has been verified for $n=2$ and $n=3$ using explicit character values [29]. In the case det $g \neq 1$, only minor modifications are required in our arguments to prove Conjecture 6.3. The spot in our proof that breaks down for regular elliptic elements with det $g=1$ is the identity (4.4). For example, when $s=n=4$ and $q=2$, if one chooses $\beta$ in $\mathbb{F}_{2^{4}}^{\times}$with $\beta^{5}=1$ (so still one has $\mathbb{F}_{2^{4}}=\mathbb{F}_{2}(\beta)$, but $\mathbb{F}_{2^{4}}^{\times} \neq\langle\beta\rangle$ ), then there are three homomorphisms $\varphi$ with free Frobenius orbits and $\sum_{\varphi}\left(\varphi(\beta)+\varphi\left(\beta^{2}\right)+\varphi\left(\beta^{4}\right)+\varphi\left(\beta^{8}\right)\right)=-3 \quad(\neq 0=\mu(4))$. Nevertheless, in this $\mathrm{GL}_{4}\left(\mathbb{F}_{2}\right)$ example it appeared from GAP [30] computations that such regular elliptic $g$ with $g^{5}=1$ had the same number of factorizations into $\ell$ reflections for all $\ell$ as did a Singer cycle in $\mathrm{GL}_{4}\left(\mathbb{F}_{2}\right)$.

Remark 6.4 On the other hand, in considering transitivity of Hurwitz actions, we did see a difference in behavior for regular elliptic elements versus Singer cycles: in $\mathrm{GL}_{4}\left(\mathbb{F}_{2}\right)$, there are $3375=\left(2^{4}-1\right)^{4-1}$ short reflection factorizations $t_{1} t_{2} t_{3} t_{4}$ both for the Singer cycles (the elements whose characteristic polynomials are $x^{4}+x^{3}+1$ or $\left.x^{4}+x+1\right)$ and for the non-Singer cycle regular elliptic elements (the elements whose characteristic polynomials are $\left.x^{4}+x^{3}+x^{2}+x+1\right)$. However, for the Singer cycles, these factorizations form one Hurwitz orbit, while for the non-Singer cycle regular elliptic elements they form four Hurwitz orbits.

\subsection{The approach of Hausel, Letellier, and Rodriguez-Villegas}

The number of factorizations $g=t_{1} t_{2} \cdots t_{\ell}$ where $t_{1}, \ldots, t_{\ell}, g$ come from specified $\mathrm{GL}_{n}\left(\mathbb{F}_{q}\right)$ conjugacy classes $C_{1}, \ldots, C_{\ell}, C_{\ell+1}$ appears in work of Hausel, Letellier, and Rodriguez-Villegas [11] and more recently Letellier [21]. They interpret it in terms of the topology of objects called character varieties under certain genericity conditions [21, Definition 3.1] on the conjugacy classes. One can check that these conditions are satisfied in the case of interest to us, that is, when $C_{\ell+1}$ is a conjugacy class of Singer cycles and the $C_{1}, \ldots, C_{\ell}$ are all conjugacy classes of reflections. Assuming 
these genericity conditions, [21, Theorem 4.14] gives an expression for the number of such factorizations in terms of a specialization $\mathbb{H}_{\omega}\left(q^{-\frac{1}{2}}, q^{\frac{1}{2}}\right)$ of a rational function $\mathbb{H}_{\omega}(z, w)$ defined in $[11, \S 1.1]$ via Macdonald symmetric functions. In principle, this expression should recover Theorem 1.3 as a very special case. However, in practice, the calculation of $\mathbb{H}_{\omega}(z, w)$ is sufficiently intricate that we have not verified it.

\subsection{Jucys-Murphy approach?}

The formulas for character values on semisimple reflections and transvections in Lemma 4.8(ii) and Corollary 4.11 are remarkably simple compared to the machinery used in their proofs. Can they be developed using a $q$-analog of the Okounkov-Vershik approach $[4,31]$ to the ordinary character theory of $\mathfrak{S}_{n}$, using the commuting family of Jucys-Murphy elements [17,24], a multiplicity-free branching rule, a Gelfand-Zetlin basis, etc.? Such a theory might even allow one to prove $q$-analogs for more general generating function results, such as one finds in Jackson [16].

A feature of the $\mathfrak{S}_{n}$ theory (see Chapuy and Stump [5, §5], Jucys [17, §4]) is that any symmetric function $f\left(x_{1}, \ldots, x_{n}\right)$ when evaluated on the Jucys-Murphy elements $J_{1}, \ldots, J_{n}$ acts as a scalar in each $\mathfrak{S}_{n}$-irreducible $V^{\lambda}$, and this scalar is $f\left(c\left(a_{1}\right), \ldots, c\left(a_{n}\right)\right)$ where $c\left(a_{i}\right)$ are the contents of the cells of $\lambda$. Taking $f=\sum_{i=1}^{n} x_{i}$ gives a quick calculation of the irreducible characters evaluated on $\sum_{i=1}^{n} J_{i}$, the sum of all transpositions. Lemma 4.8(ii) and Corollary 4.11 seem suggestive of a $q$-analog for this assertion.

It is at least clear how one might define relevant Jucys-Murphy elements.

Definition 6.5 For $1 \leq m<n$ embed $\mathrm{GL}_{m} \subset \mathrm{GL}_{n}$ as the subgroup fixing $e_{m+1}, \ldots, e_{n}$. Then, for each $\alpha \in \mathbb{F}_{q}^{\times}$, let $J_{m}^{\alpha}:=\sum_{t} t$ be the sum inside the group algebra $\mathbb{C G L}_{n}$ over this subset of reflections:

$$
\text { \{reflections } \left.t \in \mathrm{GL}_{m}: \operatorname{det}(t)=\alpha \text { and } t \notin \mathrm{GL}_{m-1}\right\} \text {. }
$$

Proposition 6.6 The elements $\left\{J_{m}^{\alpha}\right\}$ for $m=1,2, \ldots, n$ and $\alpha$ in $\mathbb{F}_{q}^{\times}$pairwise commute.

Proof Note that $J_{n}^{\alpha}$ commutes with any $g$ in $\mathrm{GL}_{n-1}$, or equivalently, $g J_{n}^{\alpha} g^{-1}=J_{n}^{\alpha}$, since conjugation by $g$ induces a permutation of the set in (6.5). This shows that $J_{n}^{\alpha}, J_{m}^{\beta}$ commute when $n \neq m$, since if one assumes $m<n$, then every term of $J_{m}^{\beta}$ lies in $\mathrm{GL}_{n-1}$. To see that $\left[J_{n}^{\alpha}, J_{n}^{\beta}\right]=0$, note that our conjugacy sums $z_{\alpha}=: z_{n, \alpha}$ from Definition 5.2 lie the center of $\mathbb{C G L}_{n}$ and can be expressed as $z_{n, \alpha}=\sum_{i=1}^{n} J_{i}^{\alpha}$. Therefore,

$$
0=\left[z_{n, \alpha}, J_{n}^{\beta}\right]=\left[\sum_{i=1}^{n} J_{i}^{\alpha}, J_{n}^{\beta}\right]=\left[J_{n}^{\alpha}, J_{n}^{\beta}\right]+\left[\sum_{i<n} J_{i}^{\alpha}, J_{n}^{\beta}\right]=\left[J_{n}^{\alpha}, J_{n}^{\beta}\right]
$$

using bilinearity of commutators, and the commutativity of $J_{i}^{\alpha}, J_{n}^{\beta}$ for $i<n$. 
Acknowledgments The authors thank A. Ram and P. Diaconis for pointing them to this work of Hildebrand [12] used in Sect. 4.4. They also thank A. Henderson and E. Letellier for pointing them to [11,21]. This work is partially supported by NSF grants DMS-1148634 and DMS-1001933.

\section{References}

1. Armstrong, D.: Generalized noncrossing partitions and combinatorics of Coxeter groups. Mem. Am. Math. Soc. 202, 949 (2009)

2. Bessis, D.: The dual braid monoid. Ann. Sci. École Norm. Sup 36(4), 647-683 (2003)

3. Bessis, D.: Finite complex reflection arrangements are $K(\pi, 1)$, arXiv preprint arXiv:math/0610777

4. Ceccherini-Silberstein, T., Scarabotti, F., Tolli, T.: Representation Theory of the Symmetric Groups. The Okounkov-Vershik Approach, Character Formulas, and Partition Algebras, Cambridge Studies in Advanced Mathematics 121. Cambridge University Press, Cambridge (2010)

5. Chapuy, G., Stump, C.: Counting factorizations of Coxeter elements into products of reflections, arXiv, preprint arXiv:1211.2789

6. Dénes, J.: The representation of a permutation as the product of a minimum number of transpositions and its connection with the theory of graphs. Publ. Math. Inst. Hung. Acad. Sci. 4, 63-70 (1959)

7. Frobenius, F.G.: Uber Gruppencharacktere (1896), in Gesammelte Abhandlungen III. Springer, Berlin (1968)

8. Garsia, A.M., Haiman, M.: A random $(q, t)$-hook walk and a sum of Pieri coefficients. J. Comb. Theory Ser. A 82, 74-111 (1998)

9. Gasper, G., Rahman, M.: Basic Hypergeometric Series, 2nd edn. Cambridge University Press, Cambridge (2004)

10. Green, J.A.: The characters of the finite general linear groups. Trans. Am. Math. Soc. 80, 402-447 (1955)

11. Hausel, T., Letellier, E., Rodriguez-Villegas, F.: Arithmetic harmonic analysis on character and quiver varieties. Duke Math. J. 160, 323-400 (2011)

12. Hildebrand, M.: Generating random elements in $S L_{n}\left(\mathbb{F}_{q}\right)$ by random transvections. J. Algebr. Comb. 1, 133-150 (1992)

13. Huang, J.: Combinatorics and invariant theory of finite general linear groups, preliminary oral exam paper. Univ. of Minnesota (2010)

14. Hurwitz, A.: Ueber Riemann'sche Flächen mit gegebenen Verzweigungspunkten, Math. Ann. 39, 1-60 (1891)

15. Jackson, D.M.: Counting cycles in permutations by group characters, with an application to a topological problem. Trans. Am. Math. Soc. 299, 785-801 (1987)

16. Jackson, D.M.: Some combinatorial problems associated with products of conjugacy classes of the symmetric group. J. Comb. Theory Ser. A 49, 363-369 (1988)

17. Jucys, A.-A.A.: Symmetric polynomials and the center of the symmetric group ring. Rep. Math. Phys. 5, 107-112 (1974)

18. Kerov, S.V.: (1992) $q$-analogue of the hook walk algorithm and random Young tableaux, Funktsional. Anal. I Prilozhen. 26(3), 35-45 (1992); translation in Funct. Anal. Appl. 26(3), 179-187

19. Lando, S.K., Zvonkin, A.K.: Graphs on Surfaces and Their Applications. Encyclopaedia of Mathematical Sciences 141. Springer, Berlin (2004)

20. Lang, S.: Algebra, Revised Third Edition, Graduate Texts in Mathematics 211. Springer, New York (2002)

21. Letellier, E.: Character varieties with Zariski closures of $G L_{n}$-conjugacy classes at punctures, arXiv, preprint arXiv:1309.7662

22. Lux, K., Pahlings, H.: Representations of Groups, Cambridge Studies in Advanced Mathematics 124. Cambridge University Press, Cambridge (2010)

23. Macdonald, I.G.: Symmetric Functions and Hall Polynomials, Oxford Mathematical Monographs, 2nd edn. Oxford Science Publications, New York (1995)

24. Murphy, G.E.: A new construction of Young's seminormal representation of the symmetric group. J. Algebra 69, 287-297 (1981)

25. Reiner, V., Stanton, D., Webb, P.: Springer's regular elements over arbitrary fields. Math. Proc. Camb. Philos. Soc. 141, 209-229 (2006) 
26. Reiner, V., Stanton, D., White, D.: The cyclic sieving phenomenon. J. Comb. Theory Ser. A 108, 17-50 (2004)

27. Silberger, A.J., Zink, E.-W.: The characters of the generalized Steinberg representations of finite general linear groups on the regular elliptic set. Trans. Am. Math. Soc. 352, 3339-3356 (2000)

28. Stanley, R.P.: Enumerative Combinatorics. vol 2, Cambridge Studies in Advanced Mathematics 62. Cambridge University Press, Cambridge (1999)

29. Steinberg, R.: The representations of $G L(3, q), G L(4, q), P G L(3, q)$, and $P G L(4, q)$. Can. J. Math. 3, 225-235 (1951)

30. The GAP Group, GAP - Groups, Algorithms, and Programming, Version 4.6.4; 2013, (http://www. gap-system.org)

31. Vershik, A.M., Okounkov, A.: A new approach to representation theory of symmetric groups. II. J. Math. Sci. (N. Y.) 131(2), 5471-5494 (2005)

32. Zelevinsky, A.V.: Representations of Finite Classical Groups: A Hopf Algebra Approach. Lecture Notes in Mathematics 869. Springer, Berlin (1981) 